\title{
Fast Logic with Slow Qubits: Microwave-Activated Controlled-Z Gate on Low-Frequency Fluxoniums
}

\author{
Quentin Ficheux $\odot,{ }^{1, *}$ Long B. Nguyen $\odot,{ }^{1, *}$ Aaron Somoroff, ${ }^{1}$ Haonan Xiong $\odot,{ }^{1}$ Konstantin N. Nesterov®, ${ }^{2}$ \\ Maxim G. Vavilov, ${ }^{2}$ and Vladimir E. Manucharyan ${ }^{1}$ \\ ${ }^{1}$ Department of Physics, Joint Quantum Institute, and Center for Nanophysics and Advanced Materials, \\ University of Maryland, College Park, Maryland 20742, USA \\ ${ }^{2}$ Department of Physics and Wisconsin Quantum Institute, University of Wisconsin-Madison, \\ Madison, Wisconsin 53706, USA
}

(Received 4 November 2020; accepted 17 March 2021; published 3 May 2021)

\begin{abstract}
We demonstrate a controlled-Z gate between capacitively coupled fluxonium qubits with transition frequencies 72.3 and 136.3 MHz. The gate is activated by a 61.6-ns-long pulse at a frequency between noncomputational transitions $|10\rangle-|20\rangle$ and $|11\rangle-|21\rangle$, during which the qubits complete only four and eight Larmor periods, respectively. The measured gate error of $(8 \pm 1) \times 10^{-3}$ is limited by decoherence in the noncomputational subspace, which will likely improve in the next-generation devices. Although our qubits are about 50 times slower than transmons, the two-qubit gate is faster than microwave-activated gates on transmons, and the gate error is on par with the lowest reported. Architectural advantages of lowfrequency fluxoniums include long qubit coherence time, weak hybridization in the computational subspace, suppressed residual ZZ-coupling rate (here $46 \mathrm{kHz}$ ), and the absence of either excessive parameter-matching or complex pulse-shaping requirements.
\end{abstract}

DOI: 10.1103/PhysRevX.11.021026

\section{INTRODUCTION}

Macroscopic superconducting circuits have emerged as a leading platform for implementing a quantum computer [1]. Currently available small-scale quantum processors [2-9] have achieved a number of important milestones, including the break-even point in quantum error correction of a single logical qubit [10], digital quantum simulation [11-16], nontrivial optimization algorithms [17-19], and an example demonstration of quantum supremacy with 53 qubits [20]. This progress is even more spectacular as it is solely based on a single qubit type, the transmon, which is essentially a weakly anharmonic electromagnetic oscillator [21]. Although the transmon's simplicity makes it a remarkably robust quantum system, its weak anharmonicity has become a major limiting factor for the current performance and scaling of quantum processors. Irrespective of implementation details, the weak anharmonicity leads to slower two-qubit gates, which makes them

\footnotetext{
*These authors contributed equally to this work.
}

Published by the American Physical Society under the terms of the Creative Commons Attribution 4.0 International license. Further distribution of this work must maintain attribution to the author(s) and the published article's title, journal citation, and DOI.

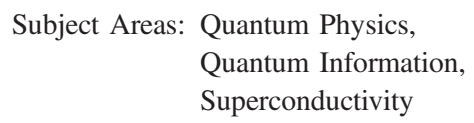

prone to decoherence errors. Therefore, a motivation has built up for exploring strongly anharmonic alternatives to transmons that would ideally have higher intrinsic coherence and be compatible with the transmon-based scaling architectures.

In recent years, coherence times in the $100 \mu$ s range were repeatedly observed in superconducting fluxonium qubits $[22,23]$. In this paper, we describe the first logical operation on a pair of capacitively coupled fluxoniums [Fig. 1(a)]. Each fluxonium can be viewed as a transmon with the weak Josephson junction additionally shunted by a large-value inductance, provided by an array of about 100 stronger junctions [24]. The inductive shunting makes the charges on the capacitors continuous, and, hence, fluxoniums can have highly anharmonic spectra insensitive to the offset charge noise. We operate fluxoniums near the half-integer flux bias ("sweet spot"), where the qubit transition frequency is first-order insensitive to flux noise and belongs to a 100-1000 MHz range. Such an order of magnitude qubit slowdown, as compared to transmons, the frequency of which is typically constrained to the $5-6 \mathrm{GHz}$ range, dramatically reduces the rate of energy relaxation due to dielectric loss and leads to long coherence times [22]. However, a fundamental question remains to be answered: How can one physically slow down qubits without ending up with slower two-qubit gates? 
(a)

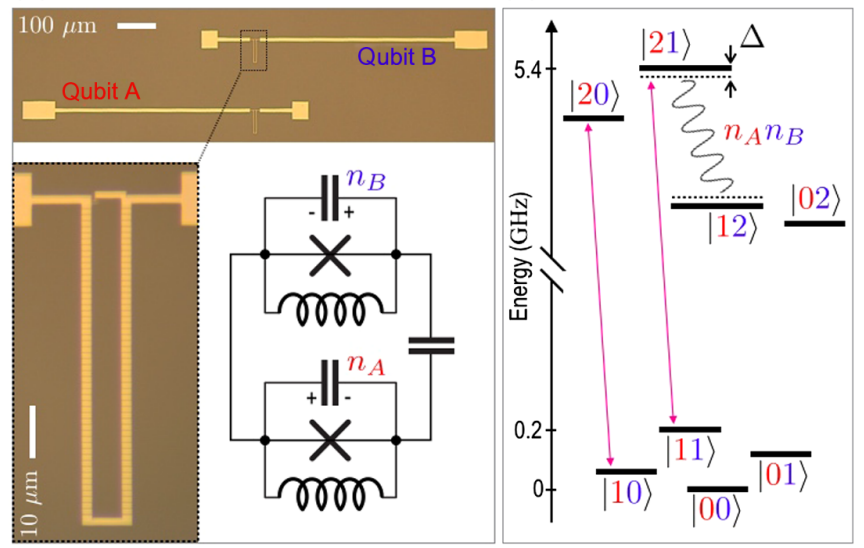

FIG. 1. (a) Optical image of two capacitively coupled fluxoniums fabricated on a silicon chip along with their minimal circuit model. Devices are similar to those reported in Ref. [22] except that the antennas are intentionally made asymmetric for optimal coupling to the readout cavity [not shown]. (b) Diagram of the lowest-energy states of the interacting two-fluxonium system. Capacitive coupling induces a shift of level $|21\rangle$ by $\Delta$ due to repulsion from level $|12\rangle$. The shift $\Delta$ enables a $\mathrm{CZ}$ operation in time approximately given by $1 / \Delta$ when either qubit is driven at a frequency in between the transitions $|10\rangle-|20\rangle$ and $|11\rangle-|21\rangle$.

Let us consider the simplest form of circuit coupling by means of a mutual capacitance. In analogy with transmons, there is a coupling term proportional to $n_{A} n_{B}$, where $n_{A}$ and $n_{B}$ are the charge operators of qubits $A$ and $B$, respectively, normalized to the Cooper pair charge $2 e$. Indeed, such a term produces little effect on the computational states $|00\rangle$, $|01\rangle,|10\rangle$, and $|11\rangle$, because the transition matrix elements of $n_{A(B)}$ vanish with the transition frequency. For the same reason, though, the much higher-energy noncomputational states $|12\rangle$ and $|21\rangle$ can experience a noticeable repulsion, while the only nearby states $|20\rangle$ and $|02\rangle$ remain unaffected due to the parity selection rule; see Fig. 1(b). Therefore, connecting the two subspaces with radiation can induce an on-demand qubit-qubit interaction. For example, Ref. [25] describes a controlled-Z (CZ) gate obtained by applying a $2 \pi$ pulse to transition $|11\rangle-|21\rangle$ while the closest transition $|10\rangle-|20\rangle$ stays unexcited. This condition can always be met if the gate pulse is much longer than $1 / \Delta$, where $\Delta$ is the shift of level $|21\rangle$ due to the $n_{A} n_{B}$ term, shown in Fig. 1(b). In fact, the CZ gate can be completed in a time close to $1 / \Delta$ by choosing the combination of drive detuning and amplitude that synchronizes the Rabi rotations of both noncomputational transitions. For our specific device parameters (see Tables I and II), we get $\Delta=22 \mathrm{MHz}(1 / \Delta=45.5 \mathrm{~ns})$, and the optimal gate time is $61.6 \mathrm{~ns}$. Remarkably, since the value of $\Delta$ is not directly tied to the qubit frequencies (here, 72.3 and 136.3 MHz), the logical operation takes just a few qubit Larmor periods.
TABLE I. Parameters of the Hamiltonian given by Eq. (1) extracted by its fitting to the two-tone spectroscopy data in Fig. 2.

\begin{tabular}{lcccc}
\hline \hline & $E_{C, \alpha}(\mathrm{GHz})$ & $E_{L, \alpha}(\mathrm{GHz})$ & $E_{J, \alpha}(\mathrm{GHz})$ & $J_{C}(\mathrm{GHz})$ \\
\hline Qubit $A$ & 0.973 & 0.457 & 5.899 & 0.224 \\
Qubit $B$ & 1.027 & 0.684 & 5.768 & \\
\hline \hline
\end{tabular}

Population transit through noncomputational states is common for gates realized with transmons [26-31]. For example, repulsion of states $|11\rangle$ and $|20\rangle$ enables a CZ gate via adiabatic flux tuning of these states in and out of resonance $[32,33]$. Recent high-fidelity versions of this gate rely on diabatic flux pulses $[28,31]$, resulting in a significant population of state $|20\rangle$ for a short time, which draws parallels to our microwave-controlled scheme. In the case of fixed-frequency qubits, repulsion between states $|03\rangle$ and $|12\rangle$ is used in Ref. [34] to implement a CZ gate activated by a microwave pulse at a frequency near the transition $|11\rangle-|12\rangle$. However, the insufficient transmon anharmonicity introduces many nearby transitions (there is only one relevant transition $|10\rangle-|20\rangle$ for fluxoniums), and in the end such a scheme proves impractical. Even in gates designed to operate entirely within the computational subspace, e.g., the flux-activated $|10\rangle-|01\rangle$ swap gate [35-37] or the cross-resonance (CR) gate [38,39], uncontrolled population leakage to noncomputational states remains an important factor limiting gate speed [40]. Yet, such coherent errors can be practically eliminated in fluxoniums owing to their highly anharmonic spectra, as exemplified by the gate scheme reported here.

Another noteworthy property of fluxoniums is that the static ZZ shift, coming from the repulsion of computational and noncomputational states, is relatively small (here, about $46 \mathrm{kHz}$ ), largely thanks to the low qubit frequencies. For transmons, the static $Z Z$ shift is an important source of gate error, the mitigation of which draws additional resources. Thus, in the case of the cross-resonance gate, the $\mathrm{ZZ}$ term is suppressed by a combination of circuit parameter matching, additional echo pulse sequences incorporated into the gate protocol [40], and additional qubit rotations [41]. An alternative strategy to eliminate the $Z Z$ shift is to use flux-tunable couplers $[20,42,43]$, which in practice act as separate quantum systems and, hence, increase the circuit complexity. More recently, the $Z Z$ shift was suppressed in capacitively shunted flux qubits using an

TABLE II. Frequencies and coherence times of transitions participating in the gate operation.

\begin{tabular}{lcccc}
\hline \hline Transition & Freq $(\mathrm{GHz})$ & $T_{1}(\mu \mathrm{s})$ & $T_{2}^{R}(\mu \mathrm{s})$ & $T_{2}^{E}(\mu \mathrm{s})$ \\
\hline$|00\rangle-|10\rangle$ & 0.07233 & 347 & 5.6 & 31 \\
$|00\rangle-|01\rangle$ & 0.13641 & 282 & 25.4 & 64 \\
$|10\rangle-|20\rangle$ & 5.1766 & 8.9 & 2.5 & 9.3 \\
$|11\rangle-|21\rangle$ & 5.1986 & 6.1 & 1.7 & 4.3 \\
\hline \hline
\end{tabular}


additional drive, but at the expense of operating away from the flux sweet spot [44].

The gate error in our scheme is largely limited by decoherence outside the computational subspace. Randomized benchmarking yields the gate error of $8 \times 10^{-3}$, which is consistent with the measured few microseconds coherence times of transitions $|10\rangle-|20\rangle$ and $|11\rangle-|21\rangle$ (see Table II). Because these transitions are transmonlike, we expect their coherence to improve by an order of magnitude in the next-generation experiments with improved fabrication and noise-filtering procedures. This step would lower the gate error into the $10^{-4}$ range. The presently achieved infidelity is on par with the lowest reported values in microwave-activated gates [41,45] (cross-resonance gate by IBM). Very recently, the CR gate error was reduced even further [46]. Additionally, our gate is considerably faster than cross-resonance-type gates with comparable errors $[40,47]$. Our result clearly illustrates the potential of highly anharmonic circuits for quantum information processing, and it motivates the exploration of large-scale quantum processors based on fluxoniums.

The paper is organized as follows: In Sec. II, we describe the details of our experimental setup, including spectroscopy of the two-fluxonium device, the joint single-shot readout of fluxoniums, and state initialization procedures. Note that the details of single-qubit gate characterization are described in the Appendix D. In Sec. III, we detail the concepts behind our fast microwave-activated CZ gate. Section IV presents the results of the CZ gate characterization, including quantum process tomography and randomized benchmarking. In Sec. V, we review the technical limitations of the present experiment and project the nearterm improvements. Section VI concludes the work.

\section{TWO-FLUXONIUM SYSTEM CHARACTERIZATION}

Our device is composed of two fluxonium artificial atoms with a circuit design introduced in Ref. [22] coupled via a shared capacitance [see Fig. 1(a)]. The system obeys the Hamiltonian [25]

$$
\begin{aligned}
\frac{\hat{H}}{h}= & \sum_{\alpha=A, B}\left[4 E_{C, \alpha} \hat{n}_{\alpha}^{2}+\frac{E_{L, \alpha}}{2} \hat{\varphi}_{\alpha}^{2}-E_{J, \alpha} \cos \left(\hat{\varphi}_{\alpha}-\phi_{\mathrm{ext}, \alpha}\right)\right] \\
& +J_{C} \hat{n}_{A} \hat{n}_{B},
\end{aligned}
$$

where $E_{C, \alpha}, E_{L, \alpha}$, and $E_{J, \alpha}$ are the charging energy, the inductive energy, and the Josephson energy of fluxonium indexed by $\alpha=A, B$, respectively. The operators $\hat{\varphi}_{\alpha}$ and $\hat{n}_{\alpha}$ are the phase twist across the inductance $L_{\alpha}$ and the charge on the capacitor $C_{\alpha}$, respectively, and they commute according to $\left[\hat{\varphi}_{\alpha}, \hat{n}_{\alpha}\right]=i$. We use spectroscopy data in order to accurately extract the parameters of the Hamiltonian (1) (see Fig. 2 and Table I).

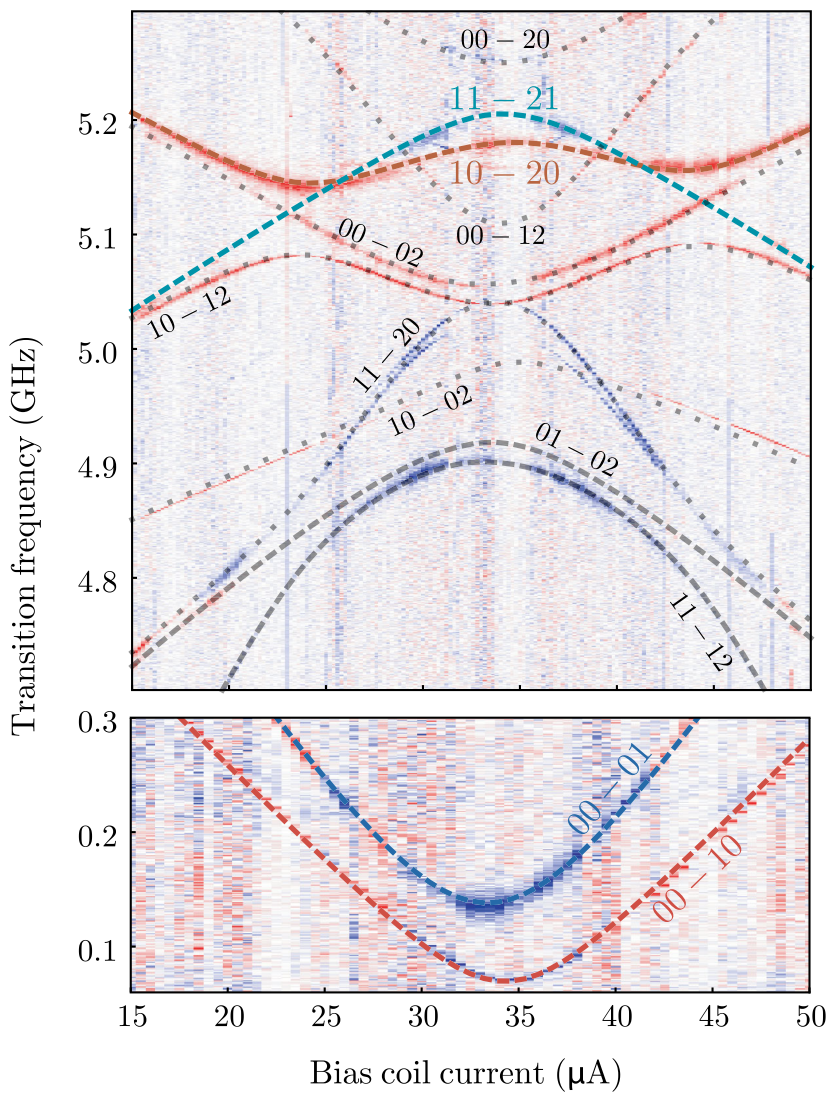

FIG. 2. Two-tone spectroscopy as a function of probe tone frequency and flux threading the common biasing coil. The parameters of the system (Table I) are extracted by fitting the spectroscopy data to the diagonalization of the Hamiltonian (1). Dashed lines represent transitions allowed at half-integer flux, while dotted lines represent transitions that are forbidden at the sweet spot. The shared capacitance lifts the degeneracy between the two transitions $|11\rangle-|21\rangle$ and $|10\rangle-|20\rangle$ by an amount $\Delta$ (top) that limits the gate speed. The sweet-spot misalignment (bottom) is attributed to a local flux imbalance through the two fluxonium loops $\phi_{\mathrm{ext}, A} \neq \phi_{\mathrm{ext}, B}$.

When biased at $\phi_{\text {ext }, \alpha}=\pi$, the fluxoniums are at their sweet spots with respect to external flux. The qubit frequencies are $f_{A}=72.3 \mathrm{MHz}$ and $f_{B}=136.3 \mathrm{MHz}$. These relatively low-frequency transitions exhibit long energy relaxation times $T_{1, A}=347 \mu \mathrm{s}$ and $T_{1, B}=282 \mu \mathrm{s}$ owing to their decoupling from dielectric loss mechanisms [22]. A slight nonuniformity in the magnetic field, provided by a single external coil, prevents biasing qubits precisely at their sweet spots simultaneously, with the offset being about $0.14 \%$ of the flux quantum. We operate at a bias coil current of $33.7 \mu \mathrm{A}$, approximately halfway between the two sweet spots, which reduces the spin echo coherence times to $T_{2, A}^{E}=31 \mu \mathrm{s}$ and $T_{2, B}^{E}=64 \mu \mathrm{s}$ (see Table II) compared to their sweet-spot values of 47 and $67 \mu \mathrm{s}$, respectively. The sweet-spot values of coherence times are likely limited by photon shot noise due to imperfect thermalization of the readout cavity [48]. This common issue can 
be improved with better filtering of the measurement lines. We note in advance that the small deviation from the sweet spots does not modify appreciably the operation of our twoqubit gates, and the qubit coherence times are not limiting the gate error.

Higher-energy states are separated from the computational states by an approximately $4.5 \mathrm{GHz}$ gap (Fig. 2, top). The capacitive coupling term $J_{c} \hat{n}_{A} \hat{n}_{B}$ in Eq. (1), with $J_{C}=224 \mathrm{MHz}$, corresponds to the coupling capacitance of about $1.2 \mathrm{fF}$. Note that similar value coupling capacitances are used in transmon-based quantum processors. The relevant interaction energy scale $\Delta$ (see Fig. 1 ) is given by $\Delta=\left|E_{20}+E_{11}-E_{10}-E_{21}\right| / h=22 \mathrm{MHz}$, and the static $Z Z$ shift is given by $\xi_{Z Z}=\left|E_{00}+E_{11}-E_{01}-E_{10}\right| / h=$ $46 \mathrm{kHz}$, where $E_{i j}$ is the energy of state $|i j\rangle$.

The device is embedded in a three-dimensional copper cavity with a resonant frequency of $f_{c}=7.4806 \mathrm{GHz}$. Prior to each experiment, the qubits are initialized by applying a strong microwave pulse on the cavity (see Appendix C 3). This procedure prepares each qubit in a state close to the excited state. After the initialization pulse, each qubit is in a mixed state with excited states probabilities of $86 \%$ and $88 \%$ for qubits $A$ and $B$, respectively, which is sufficient for metrology of gate operations. The remaining qubit entropies correspond to temperatures of $T_{A}=3.7 \mathrm{mK}$ and $T_{B}=5.9 \mathrm{mK}$ well below the fridge temperature of $14 \mathrm{mK}$. Potentially more efficient initialization procedures have been demonstrated on singlefluxonium devices $[23,49,50]$. We achieve a single-shot joint readout of the two qubits by sending a cavity tone of optimal frequency, power, and duration. The outgoing signal is further amplified by a Josephson traveling wave parametric amplifier (JTWPA) [51] and commercial amplifiers before down-conversion and numerical demodulation. We finally correct for readout imperfections by a procedure described in Appendix C 2 .

\section{FAST CZ GATE BY EXACT LEAKAGE CANCELLATION}

In this section, we describe how to implement a $\mathrm{CZ}$ gate in the case of a two-qubit spectrum shown in Fig. 1(b) in the shortest possible time. Let us start by considering the gate transitions $|10\rangle-|20\rangle$ and $|11\rangle-|21\rangle$ as two-level systems. Our gate exploits the geometric-phase accumulations during round-trips in these two systems. The accumulated phase can be divided into two parts: the dynamical phase, which is proportional to the evolution time and the energy of the system, and the geometric phase, which depends only on the trajectory followed in the Hilbert space. Applying a microwave tone drives the system with the Hamiltonian

$$
\frac{\hat{H}_{\text {drive }}}{h}=\left(\epsilon_{A} \hat{n}_{A}+\epsilon_{B} \hat{n}_{B}\right) \cos \left(2 \pi f_{d} t\right) .
$$

When the drive frequency $f_{d}$ is nearly resonant with one of the two gate transitions, we observe Rabi oscillations [Fig. 3(a)] with the resonance Rabi frequencies $\Omega_{10-20}=$ $\left|\left\langle 10\left|\varepsilon_{A} \hat{n}_{A}+\varepsilon_{B} \hat{n}_{B}\right| 20\right\rangle\right|$ and $\Omega_{11-21}=\left|\left\langle 11\left|\varepsilon_{A} \hat{n}_{A}+\varepsilon_{B} \hat{n}_{B}\right| 21\right\rangle\right|$, respectively. A strong hybridization of the $|12\rangle$ and $|21\rangle$ states creates an imbalance between the rotation speeds given by the ratio $r=\Omega_{11-21} / \Omega_{10-20} \simeq 1.36$.

In order to eliminate leakage to higher states, one needs to synchronize off-resonance Rabi oscillations determined by the two transitions to ensure that the state vector always comes back to the computational subspace. This synchronization is achieved by matching the generalized Rabi frequencies

$$
\Omega=\sqrt{\Omega_{11-21}^{2}+\delta^{2}}=\sqrt{\Omega_{10-20}^{2}+(\delta-\Delta)^{2}},
$$

where $\delta=f_{11-21}-f_{d}$ is the detuning between the $|11\rangle-|21\rangle$ transition and the drive frequency [Fig. 3(a)]. A full rotation is then performed in the shortest gate time $t_{\text {gate }}=1 / \Omega$. During the gate operation, the state vector trajectory can be depicted in a Bloch sphere representation [Fig. 3(b)] when the system starts in $|10\rangle$ or $|11\rangle$. These circular trajectories, which travel in opposite directions with respect to the centers of the Bloch spheres, define two cones inside the spheres. The cones and directions of travel define the solid angles $\Theta_{10}=2 \pi[1-(\Delta-\delta) / \Omega]$ and $\Theta_{11}=2 \pi(1+\delta / \Omega)$, which correspond to a geometric phase accumulation $\varphi_{i j}=-\Theta_{i j} / 2$ on state $|i j\rangle$. Our gate, thus, implements a unitary operation $U=\operatorname{diag}\left(1,1, e^{i \varphi_{10}}\right.$, $\left.e^{i \varphi_{11}}\right)$. Using virtual $\mathrm{Z}$ rotations [52], the phase difference can be assigned to any computational state such as $|11\rangle$ to realize a controlled-phase operation $U=\operatorname{diag}\left(1,1,1, e^{i \Delta \varphi}\right)$ with $\Delta \varphi=\varphi_{11}-\varphi_{10}$. A CZ gate is obtained when $\Delta \varphi=-\left(\Theta_{11}-\Theta_{10}\right) / 2=-\pi \Delta / \Omega= \pm \pi$. Using this condition in Eq. (3), we obtain the optimal drive frequency [brown arrow in Fig. 3(a)]

$$
\frac{\delta}{\Delta}=\frac{r^{2}-\sqrt{\left(r^{2}-1\right)^{2}+r^{2}}}{r^{2}-1} \simeq 0.29
$$

For $\delta$ given by Eq. (4) and $\Omega=\Delta$, a CZ gate with zero leakage is achieved in time exactly $t_{\text {gate }}=1 / \Delta$.

Our understanding of the gate process is validated by simulating the Hamiltonian Eq. (1) in the presence of the drive term, given by Eq. (2). The full Hamiltonian takes into account the dynamical phase $\phi_{\text {dyn }}=2 \pi \xi_{Z Z} t_{\text {gate }} \simeq 10^{-2} \ll$ $\pi$, which is negligible after one gate operation thanks to the small ZZ-interaction term. Starting with the driving frequency given by Eq. (4), we minimize the final population leakage out of the computational subspace by adjusting the drive amplitude and frequency for every gate duration. Because of the rising and lowering edges of the pulse in the simulation, we find a slightly longer optimal gate duration of approximately $62 \mathrm{~ns}$ (compared to 


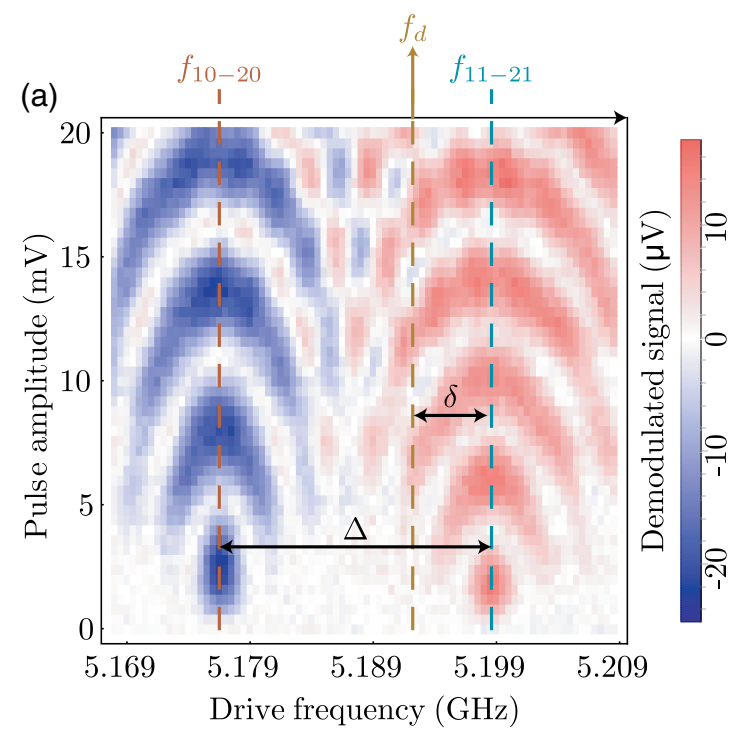

(b)

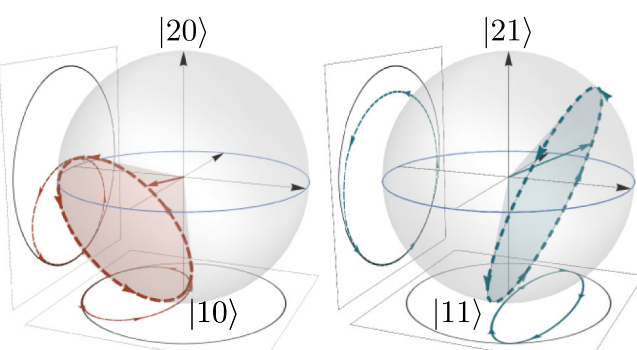

(c)

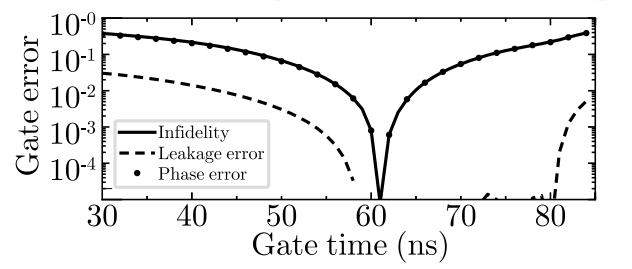

FIG. 3. Gate principle. (a) Rabi oscillations near the $|10\rangle-|20\rangle$ and $|11\rangle-|21\rangle$ transitions versus driving frequency with a 330-nslong pulse. The two transitions display different resonance Rabi frequencies characterized by the ratio $r=\Omega_{11-21} / \Omega_{10-20} \simeq 1$.36. The drive frequency $f_{d}$ indicated in brown given by Eq. (4) is used to synchronize the oscillations on the two transitions. (b) Bloch sphere representations of the trajectories in the $\{|10\rangle,|20\rangle\}$ and $\{|11\rangle,|21\rangle\}$ manifolds in the frame rotating at the drive frequency. The quantum state follows a closed path in the Hilbert space leading to a relative phase accumulation given by the difference of the solid angles spanned by the two trajectories. (c) Gate error versus gate duration simulated using the Hamiltonian given by Eq. (1) in the presence of a Gaussian flat-topped pulse and without decoherence. For every gate duration, drive frequency and amplitude are optimized to minimize the coherent leakage error (dashed line). The dots represent the gate error caused by incorrect phase accumulation $\Delta \varphi=\varphi_{11}-\varphi_{10} \neq \pi$. With the optimal pulse duration around approximately $62 \mathrm{~ns}$, the infidelity due to coherent errors is well below $10^{-3}$.

$1 / \Delta=45.5 \mathrm{~ns})$ for which coherent errors on the gate fall below $<10^{-3}$; see Fig. 3(c). Note that, even at the error level of $10^{-4}$, the optimal point does not require an excessive fine-tuning of the gate pulse parameters: It corresponds to a fraction of a percent variation in terms of the gate time, gate pulse amplitude, and frequency (see Appendix F).

\section{GATE CALIBRATION AND METROLOGY}

We start with the following initial gate parameters: We use a 60 ns flattop Gaussian pulse with a driving amplitude corresponding to a full rotation at the gate frequency given by Eq. (4). To measure the accumulated phase difference $\Delta \varphi$, we perform a Ramsey-type experiment on qubit $B$ to compare the phases of the superpositions $|11\rangle+|10\rangle$ and $|01\rangle+|00\rangle$ after the application of a $\mathrm{CZ}$ gate. This comparison is achieved by inserting a $\mathrm{CZ}$ gate between two successive $\pi / 2$ pulses with a relative phase $\beta$ created by a virtual $\mathrm{Z}$ rotation [52]. When the angle $\beta$ is varied, we observe Ramsey fringes with an initial phase encoding the excited state probability of the control qubit $A$. The phase difference when qubit $A$ is prepared in the ground or excited state yields the relative phase accumulation $\Delta \varphi / \pi \simeq 0.95$ [in Fig. 4(a)] close to the phase required for the $\mathrm{CZ}$ gate.

In order to gain more insight in the physics of our gate, we perform quantum process tomography by preparing 16 independent input states, applying the $\mathrm{CZ}$ gate to each of them before performing their state tomography. The state tomography is obtained by a maximum likelihood estimation similar to the one in Ref. [53] using an overcomplete set of 36 tomography pulses. We represent the quantum process [54] in the Pauli basis through the process tomography matrix $\chi[47,55]$ [Fig. 4(b)] and adjust the single-qubit phases with virtual $\mathrm{Z}$ rotations to attribute the relative phase accumulation to the $|11\rangle$ state only. We obtain process fidelity $F_{\mathrm{QPT}}=0.97$. Note that, although our input states are not pure quantum states, our procedure is valid, since we prepare a set of independent input states. This result shows that our scheme can be used to implement a $\mathrm{CZ}$ gate, but the value of the fidelity is probably dominated by state preparation and measurement (SPAM) errors (Appendixes C 2 and D) and cannot be used to optimize the gate parameters further.

We turn to randomized benchmarking $(\mathrm{RB})$ to obtain a finer estimate of the gate error. The $\mathrm{RB}$ sequence is composed of $m$ randomly chosen Clifford operations followed by a recovery gate aimed at bringing the quantum state back to the initial state. The average population of $|11\rangle$ state decays as $a+b p^{m}+c(m-1) p^{m-2}$, where $m$ is the number of Clifford operations, $p$ is the depolarizing parameter, and $a, b$, and $c$ are fitting parameters used to absorb state preparation and measurement errors [56]. The average fidelity of a Clifford operation is given by 
(a)

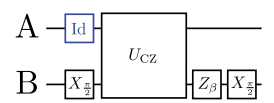

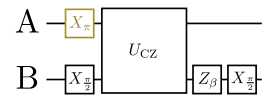
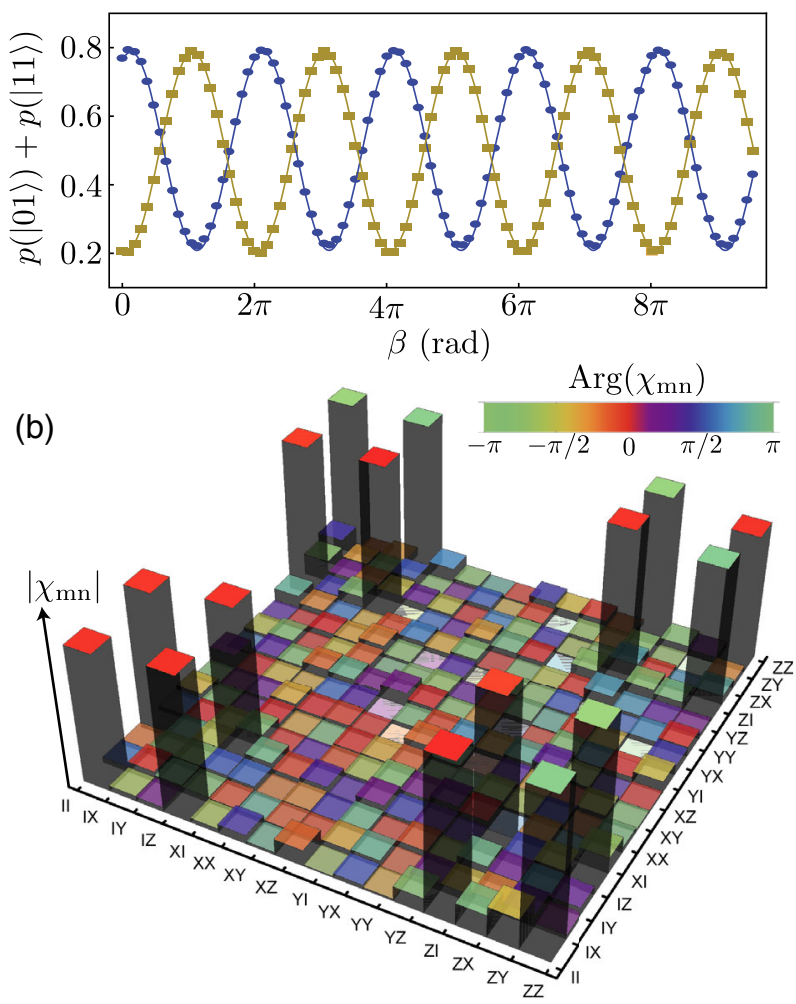

FIG. 4. Controlled-Z gate. (a) Pulse sequences of a Ramsey-type experiment measuring the phase of qubit $B$ after a $\mathrm{CZ}$ operation. Ramsey fringes are obtained by varying the rotation angle $\beta$. The excited state population of qubit $B, p_{1}^{B}=p(|01\rangle)+p(|11\rangle)$, oscillates with $\beta$ with a phase that depends on the state of the control qubit $A$. The blue and brown fringes are obtained by flipping the state of qubit $A$ at the beginning of the sequence, revealing the conditional phase accumulated during the gate $\Delta \varphi \simeq 0.95 \pi$. (b) Quantum process tomography of the CZ gate. The experimentally extracted process tomography matrix $\chi$ reproduces a CZ operation with a fidelity of 0.97 .

$F_{\text {Clifford }}=1-(d-1)(1-p) / d$, where $d=2^{n}$ is the dimension of the Hilbert space with $n=2$ the number of qubits. Interleaving a target gate yields a decay with depolarizing parameter $p_{\text {gate }}$ and a gate error of $1-F=$ $(d-1)\left(1-p_{\text {gate }} / p\right) / d$.

To reach the optimal gate fidelity, we perform a parameter search optimizing the sequence fidelity of fixed length interleaved randomized sequences [56] using a method known as "optimized randomized benchmarking for immediate tune-up" (ORBIT) [57] with a covariance matrix adaptation evolution strategy (CMA-ES) [58]. In practice, we first measure an interleaved randomized benchmarking curve [see Fig. 5(b)] before fixing the number of gates to $n=$ floor $[-1 / \log (p)]=5$, providing the optimal sensitivity. The survival probability $p(|11\rangle)$ is maximized by adjusting the six gate parameters [Fig. 5(a)]:
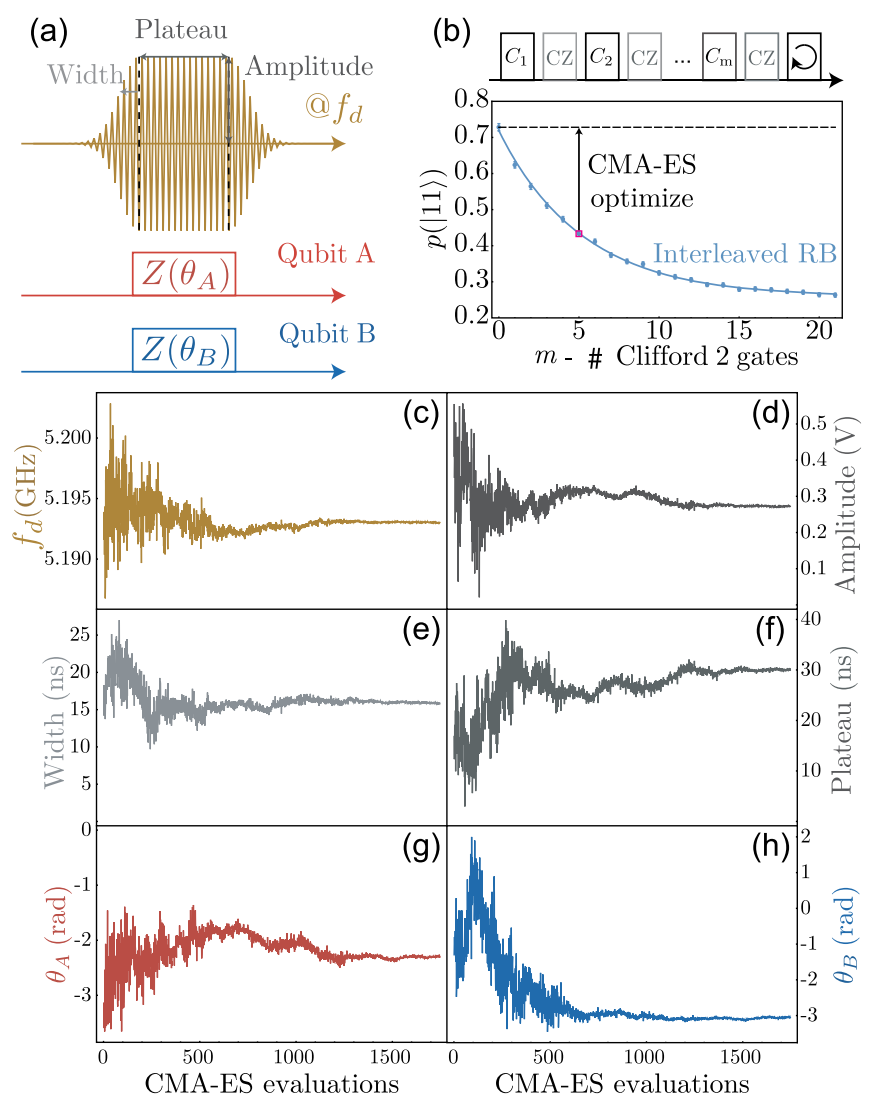

FIG. 5. Optimizing the CZ gate. (a) The gate is optimized over six parameters: pulse width, plateau, amplitude, and frequency as well as single-qubit $\mathrm{Z}$ rotation angles. (b) We use an approach based on ORBIT to optimize the sequence fidelity at fixed length with a CMA ES optimization. The number of Clifford gates [here, $5 \simeq-1 / \log (p)]$ is chosen to maximize the sensitivity of the tuneup procedure. (c)-(h) Stochastic evolution of the gate parameters versus the number of function evaluation. The fidelity of the $\mathrm{CZ}$ gate is improved from 0.967 to 0.992 .

amplitude, duration, width of the edges, frequency, and single-qubit rotation angles. Figures $5(\mathrm{c})-5(\mathrm{~h})$ show the stochastic evolution of gate parameters during 1700 search steps leading to an improvement of gate fidelity from 0.967 to 0.992 for final gate duration of $30.0($ plateau $)+2 \times 15.8$ (edges $)=61.6$ ns. In our experiment, CMA evolution strategy leads systematically to the same (global) minimum contrary to a nonstochastic algorithm such as the Nelder-Mead method that gets more easily trapped in local minima.

We assess the quality of the optimized $\mathrm{CZ}$ gate by iterative interleaved randomized benchmarking [56]. We obtain a reference fidelity $F_{\text {Clifford2 }}=(96.0 \pm 0.1) \%$. Interleaving the gate $\mathrm{CZ}^{n}$, obtained by concatenating $n$ pulses corresponding to a single $\mathrm{CZ}$ gate, yields the $\mathrm{CZ}$ gate fidelity of $F(\mathrm{CZ})=(99.2 \pm 0.1) \%$ for $n=1$ [yellow curve in Fig. 6(a)]. The error grows linearly with $n$ (we try $n=2,3, \ldots, 10$ ) [Fig. 6(b)], which is a solid evidence that the gate error is due to incoherent processes [59]. 


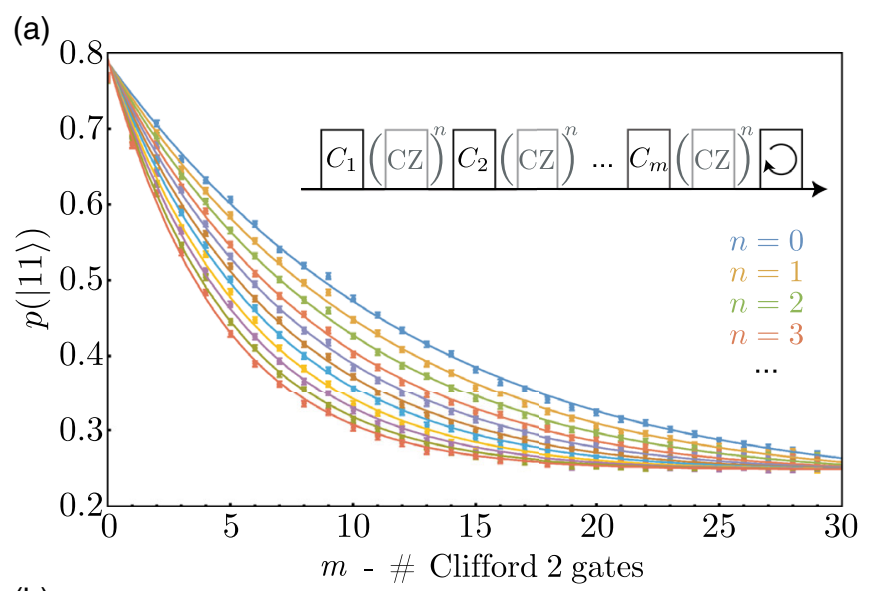

(b)

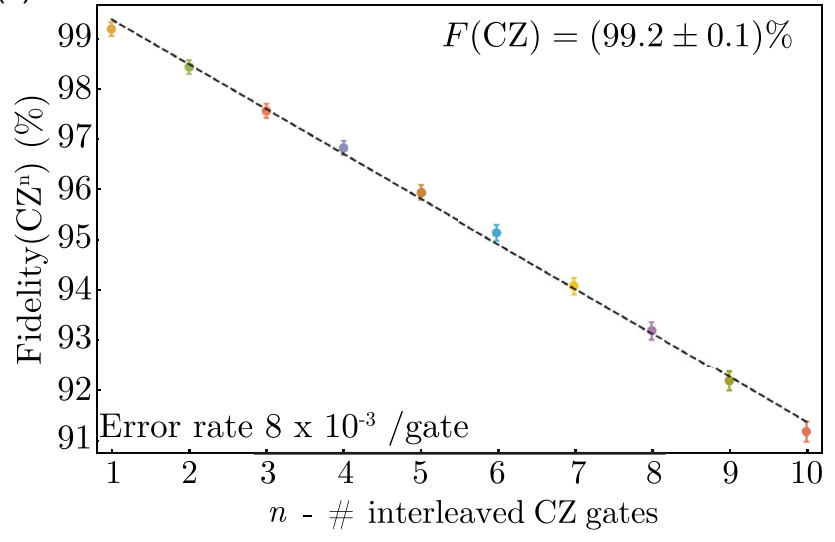

FIG. 6. Interleaved randomized benchmarking. (a) Average probability $p(|11\rangle)$ as a function of the sequence length. For each sequence length, we average over 100 random sequences repeated 1500 times. We insert a variable number $n=0,1, \ldots, 10$ (encoded in the color) of $\mathrm{CZ}$ gates between the randomly chosen Clifford gates. (b) Fidelity of $\mathrm{CZ}^{n}$ versus $n$. The gate error grows linearly with $n$, indicating that the errors are incoherent. Error bars are calculated according to the procedure given in Appendix E.

\section{OUTLOOK}

Although the benchmarked fidelity of our $\mathrm{CZ}$ gate is already high, the current experimental setup contains a number of imperfections, most of which can be eliminated in the next-generation experiments. Let us start the discussion by briefly summarizing these imperfections.

Taking into account the Ramsey coherence times of transitions $|10\rangle-|20\rangle$ and $|11\rangle-|21\rangle$ (see Table II), one expects the gate error bounded by $7.5 \times 10^{-3}$ (see Appendix F 2). This estimate falls close to the measured gate error $(8 \pm 1) \times 10^{-3}$. We believe that the limited coherence time of transitions involving the second excited state originates in the first-order sensitivity to external flux noise caused by the sweet-spot misalignment. This effect can be corrected by either improving magnetic field uniformity, likely through better magnetic shielding, or by using two independent coils. Importantly, fluxonium's $|1\rangle-|2\rangle$ transition is very similar in terms of decoherence mechanisms to a transmon qubit transition [22], and we, therefore, expect no fundamental obstacles in reaching coherence times around 50-100 $\mu \mathrm{s}$ in future work. In this case, the Hamiltonian simulations presented in Fig. 3 show that coherent gate errors in the low $10^{-4}$ range are possible for our device parameters and without sophisticated pulse optimization.

In the computational subspace, coherence times can potentially exceed $500 \mu \mathrm{s}$, given the measured energy relaxation times of $250-350 \mu$ s of states $|10\rangle$ and $|01\rangle$. Indeed, coherence time in the few hundred microseconds range was previously observed in single-fluxonium experiments. However, here, the qubit coherence time is only 50-60 $\mu$ s, even at their flux sweet spots, which can be explained by the presence of about $5 \times 10^{-3}$ thermal photons, on average, in the readout resonator. Coherence time of the gating transitions $|10\rangle-|20\rangle$ and $|11\rangle-|21\rangle$ will also be eventually limited by the shot noise of thermal photons in the readout mode. This dephasing source is generic to superconducting circuit experiments, and it can be mitigated in the future with improved cryogenic filtering of the measurement lines [60,61].

Turning to our microwave packaging choice, we use a single input port in a single 3D-box resonator to perform all the gates, for the sake of technical simplicity (see Appendix B). In Appendix D, we characterize the reduction of addressability in the system and find that simultaneous single-qubit gates have an about 3\% error rate dominated by classical cross talk. Using a dedicated driving port per qubit would enable selective addressing of each qubit and, hence, improve significantly the single-qubit gate fidelity [62]. More generally, individual addressing and readout is essential for scaling beyond the two-qubit experiments. We believe that scaling of our gate scheme can be done by moving to the 2D-circuit designs, in complete analogy with processors based on capacitively coupled transmons [2-4]. Note that our experiment does not benefit from the usually high-quality factors of 3D resonators, and, hence, it is compatible with a traditional 2D-circuit technology without conceptual modifications. The only foreseen price of replacing fixed-frequency transmons by optimally biased fluxoniums would be the requirement of either a highly homogeneous global magnetic field or a static flux-bias line per qubit.

A final remark is that our $\mathrm{CZ}$ gate does not require excessive parameter matching, unlike the majority of microwave-activated gates $[34,63]$. For example, the level diagram in Fig. 1 has no fine-tuned transitions, and the described gate protocol would work for a large class of fluxonium spectra and interaction strengths. Essentially, in order to obtain $\Delta$ in the $10-20 \mathrm{MHz}$ range, it is sufficient to arrange the energy of states $|12\rangle$ and $|21\rangle$ not far from each other (around $300 \mathrm{MHz}$ difference here) and that qubit frequencies are well resolved (about $70 \mathrm{MHz}$ here). 
This property can mitigate the effects of fabrication imperfections and improve qubit connectivity in the large-scale quantum processors.

\section{CONCLUSIONS}

We demonstrated that fluxonium qubits are not only good at storing quantum information, but they also allow for fast and high-fidelity logical operations with a minimal engineering overhead. Our implementation of the microwave-activated $\mathrm{CZ}$ gate can be decomposed into two ideas applicable to other quantum systems, each harnessing the strong anharmonicity of fluxoniums. First, the energy scale $\Delta$ limiting the gate speed comes from the repulsion of noncomputational states (here, the repulsion of states $|21\rangle$ and $|12\rangle$ ), and, hence, the gate time of approximately $1 / \Delta$ can be made, in principle, shorter than the qubit's Larmor periods. Second, by synchronizing Rabi rotations of the only two relevant noncomputational transitions (here, $|10\rangle-|20\rangle$ and $|11\rangle-|21\rangle$ ), a conditional geometric phase is accumulated at the end of each Rabi period with zero leakage outside the computational manifold. Notably, the synchronization is possible with a single microwave pulse of proper amplitude, frequency, and duration, applied to either qubit.

While the currently achieved combination of the $\mathrm{CZ}$ gate time $(61.6 \mathrm{~ns})$ and infidelity $\left(8 \times 10^{-3}\right)$ is already competitive with gates on transmons, our analysis indicates that the error can be reduced by an order of magnitude on improving the fabrication procedures, magnetic shielding, and line filtering. Given the general compatibility of our fluxonium capacitive coupling scheme with the transmonbased scaling technology, we believe that all necessary demonstrations have been made to start exploring largerscale fluxonium-based processors.

\section{ACKNOWLEDGMENTS}

We thank Chen Wang, Benjamin Huard, and Ivan Pechenezhskiy for useful discussions and acknowledge the support from NSF PFC at JQI (No. 1430094) and from ARO-LPS HiPS program (No. W911NF-18-1-0146). V.E. M. and M. G. V. acknowledge the Faculty Research Award from Google and fruitful conversations with the members of the Google Quantum AI team.

\section{APPENDIX A: DEVICE DESIGN AND FABRICATION}

The device is fabricated by standard $e$-beam lithography of a resist bilayer (MAA/PMMA) deposited on a highresistivity silicon substrate. Two layers of aluminum (20 and $40 \mathrm{~nm}$ ) [22] separated by a thin barrier of oxide are deposited by double-angle evaporation. Each inductance is composed of 310 (qubit $A$ ) and 206 (qubit $B$ ) identical Josephson junctions (in agreement with the extracted parameters $E_{L, B} / E_{L, A} \simeq 206 / 310$ ). The loops are closed by a Josephson junction whose insulating barrier parameters determine $E_{J, A(B)}$. The antennas are designed to provide a charging energy $E_{C, A}, E_{C, B} \simeq 1 \mathrm{GHz}$ required to bring the second excited state transitions in the range $4-5 \mathrm{GHz}$. The asymmetry in the antenna design enables us to independently adjust the charging energy and the coupling to the cavity mode.

Table II gives the value of the energy relaxation and coherence times of the selected transitions of the system. All the lifetimes and coherence times are measured by energy relaxation, Ramsey, and spin echo sequences. The reduced coherence time of the $|10\rangle-|20\rangle$ and $|11\rangle-|21\rangle$ is limiting the gate fidelity (see the main text).

\section{APPENDIX B: MEASUREMENT SETUP AND WIRING}

Qubit pulses are directly generated by an arbitrary waveform generator (see Fig. 7) instead of modulating a radio frequency tone. Our ability to multiplex and synthesize qubit pulses with a single digital-to-analog converter significantly reduces the hardware cost of the experiment. We use the internal mixing and pulse modulation capabilities of two Rhode and Schwarz ${ }^{\circledR}$ SMB100A sources to generate the readout pulse and the two qubit operations. The output signal is amplified by a JTWPA provided by Lincoln Labs [51] followed by commercial amplifiers before heterodyne detection.

\section{APPENDIX C: INITIALIZATION AND READOUT}

\section{Single-shot readout}

Both fluxoniums are strongly coupled to a 3D cavity. We perform a joint readout $[33,64]$ of the qubit states. In the dispersive regime [65], the readout operator can be written

$$
\hat{M}=\beta_{I Z} \mathbb{I} \otimes \sigma_{Z}+\beta_{Z I} \sigma_{Z} \otimes \mathbb{I}+\beta_{Z Z} \sigma_{Z} \otimes \sigma_{Z},
$$

where $\beta_{i j}$ are complex coefficients and $\sigma_{i}$ are Pauli matrices. In the single-shot limit, the integrated heterodyne signal distribution can be modeled as the sum of four Gaussian distributions associated with the computational states $|00\rangle,|10\rangle,|01\rangle$, and $|11\rangle$ (see Fig. 8).

We observe that the populations (Fig. 8) extracted from fitting the readout distribution by four Gaussians are affected by the readout amplitude and duration, indicating that our readout scheme is not quantum nondemolition [66-68]. Figure 9 represents the evolution of the population with the duration of a cavity pulse preceding the readout pulse. The origin of this effect will be investigated in more detail in future studies.

\section{Readout cross-talk compensation}

We adopt an empirical readout cross-talk compensation introduced in Ref. [37]. This approach is used to compensate for incorrect state mapping during a bifurcation 


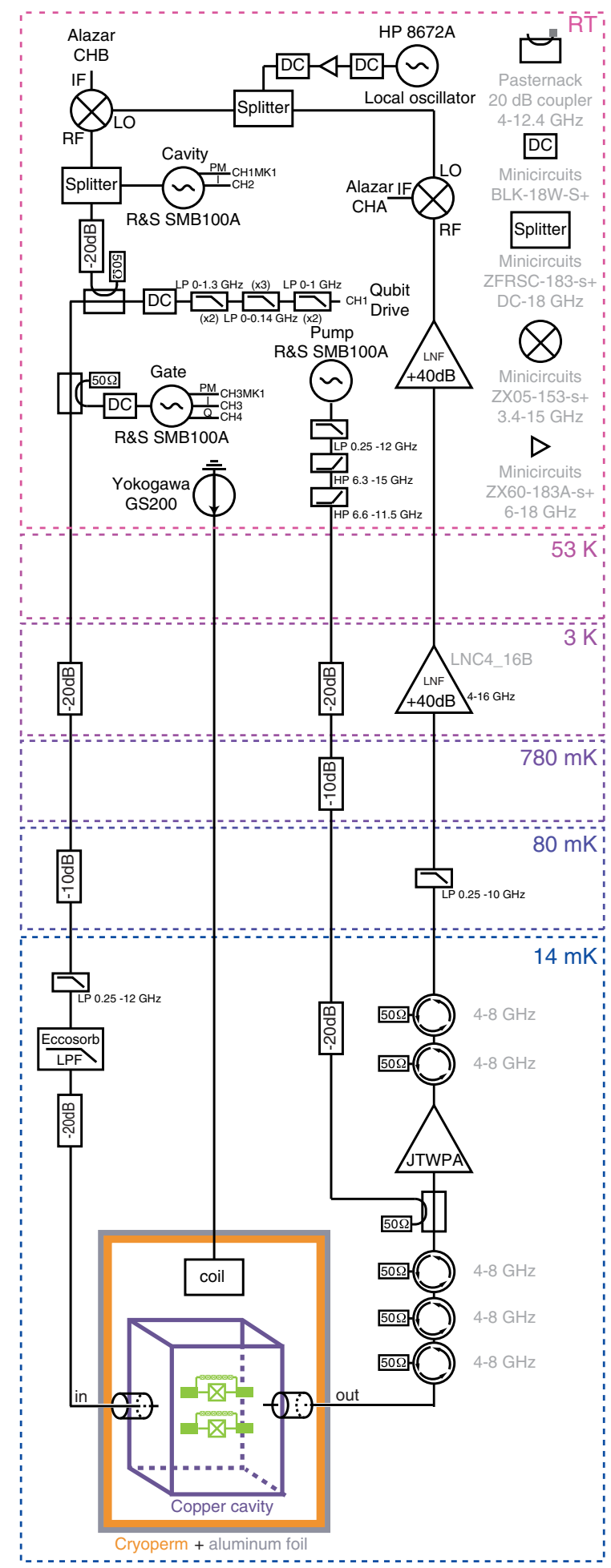

FIG. 7. Schematics of the experimental setup. Single-qubit pulses are generated directly at the qubit frequencies using one analog output port of a Tektronix ${ }^{\circledR}$ arbitrary waveform generator AWG5014C (not represented). The signal is combined with CZ gate pulses and readout pulses before reaching the input port of the $3 \mathrm{D}$ cavity. The outgoing signal is amplified using a traveling wave parametric amplifier followed by cryogenic and room temperature amplifiers before down-conversion by a local oscillator, digitization by an Alazar ${ }^{\circledR}$ acquisition board (not represented), and numerical demodulation.

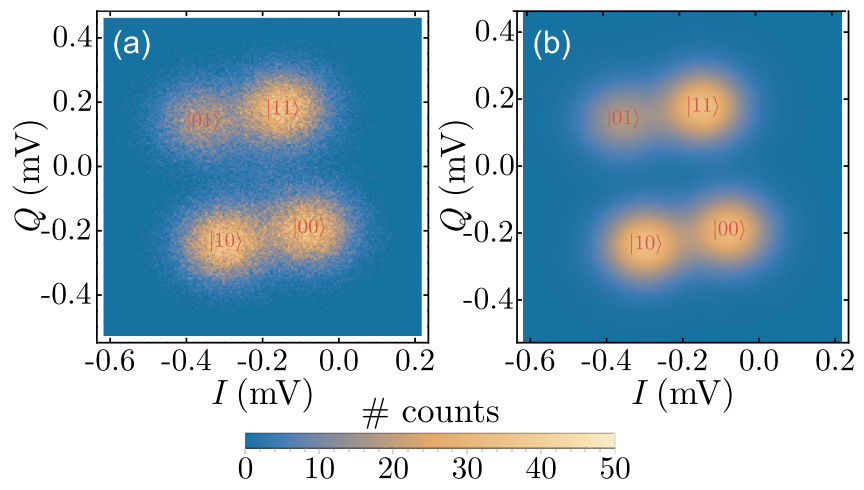

FIG. 8. Experimental (a) and fitted (b) single-shot histograms of the readout signal when the two qubits are in thermal equilibrium. Histograms in (a) are calculated from $1.5 \times 10^{5}$ experimental realizations with a $10-\mu$ s-long readout pulse. The fitting function is the sum of four Gaussian distributions. This measurement is used to calibrate the position of the center of the Gaussian distributions.

readout, but we believe that it is relevant in our case. First, we compensate for an incorrect mapping $|0\rangle \rightarrow|1\rangle$ or $|1\rangle \rightarrow|0\rangle$ of qubit $\alpha$ by correcting the qubit population of states $|i j\rangle$ with a tensorial products of two $2 \times 2$ matrices $C_{A} \otimes C_{B}$ where

$$
C_{\alpha}=\left(\begin{array}{cc}
a_{\alpha} & 1-b_{\alpha} \\
1-a_{\alpha} & b_{\alpha}
\end{array}\right)
$$

where $a_{A(B)}=0.98(0.96)$ and $b_{A(B)}=0.96(0.87)$.

In addition, we use a pure cross-talk matrix which takes into account the possibility to swap excitations between the qubits during the readout process. The total readout correction applied to the qubit populations reads

$$
\left(\begin{array}{l}
p_{00}^{\prime} \\
p_{10}^{\prime} \\
p_{01}^{\prime} \\
p_{11}^{\prime}
\end{array}\right)=\left(\begin{array}{cccc}
1 & 0 & 0 & 0 \\
0 & 1-b & c & 0 \\
0 & b & 1-c & 0 \\
0 & 0 & 0 & 1
\end{array}\right)\left(C_{A} \otimes C_{B}\right)\left(\begin{array}{c}
p_{00} \\
p_{10} \\
p_{01} \\
p_{11}
\end{array}\right),
$$

where $p_{i j}^{\prime}$ are the corrected qubit populations, $b=7 \%$, and $c=3.5 \%$. The impact of this calibration is best exemplified when performing Rabi experiments. After the calibration (Fig. 10, right column), we observe that the oscillations are centered around 0.5 (center of the Bloch sphere) and only the targeted qubit displays oscillations. Finally, we remind the reader that the two-qubit gate fidelities quoted in the main text are not affected by this procedure, since randomized benchmarking is not sensitive to readout errors [69]. 


\section{Initialization}

One may be concerned in working with qubits whose frequency is lower than the temperature because of the large initial excited state population as well as a modest reduction of the energy relaxation time. However, this situation routinely takes place in other systems, e.g., NMR. In fact, even high-frequency transmon qubits require an efficient reset procedure [70,71]. Recent experiments show a highfidelity reset of single-fluxonium qubits using measurementbased feedback [50] and sideband transitions $[23,49]$.

We describe how we turn the qubit transitions induced by cavity photons observed in Fig. 9 to our advantage to initialize the system. This shortcut is convenient for a coarse initialization procedure for a two-qubit system without any additional hardware. The rate of these transitions is known to generally increase with the number of circulating photons in the cavity $[72,73]$. We, thus, expect to be able to induce incoherent transition rates between the qubit states.

We model the photon-induced relaxation using the incoherent rate equations

$$
\begin{aligned}
& \frac{d p_{0}^{\alpha}}{d t}=-\Gamma_{\uparrow}^{\alpha} p_{0}^{\alpha}+\Gamma_{\downarrow}^{\alpha} p_{1}^{\alpha}, \\
& \frac{d p_{1}^{\alpha}}{d t}=\Gamma_{\uparrow}^{\alpha} p_{0}^{\alpha}-\Gamma_{\downarrow}^{\alpha} p_{1}^{\alpha},
\end{aligned}
$$

where the total excitation and deexcitation rates are $\Gamma_{\uparrow}^{A}=$ $38.6 \pm 0.6 \mathrm{kHz}, \quad \Gamma_{\downarrow}^{A}=6.4 \pm 0.6 \mathrm{kHz}$ and $\Gamma_{\uparrow}^{B}=53.8 \pm$ $0.3 \mathrm{kHz}, \quad \Gamma_{\downarrow}^{B}=7.4 \pm 0.3 \mathrm{kHz}, \quad$ respectively. Notably, increasing the number of circulating photons by a factor of $2.3^{2} \simeq 5$ (from Fig. 9 to Fig. 11) increases the energy

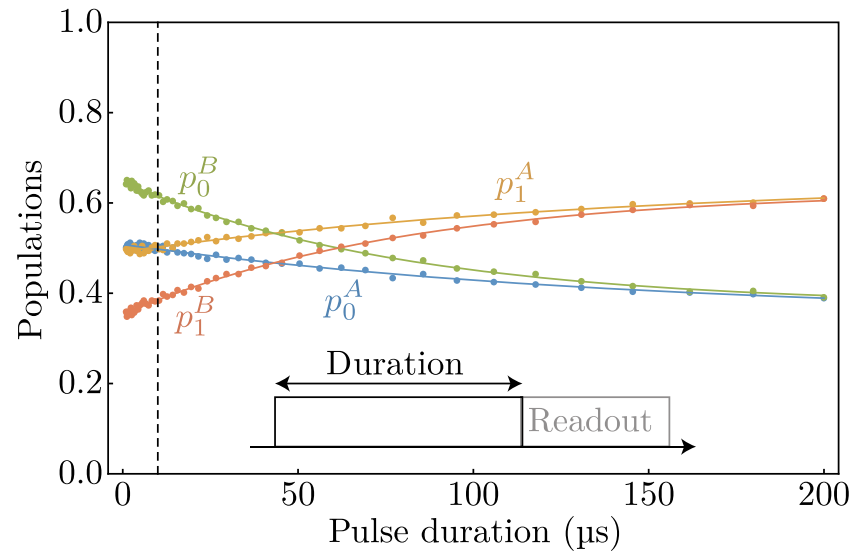

FIG. 9. Evolution of the qubit populations after a cavity pulse as a function of the pulse duration. The solid lines correspond to the solution of rate equation (C3) for each qubit with the transition rate $\Gamma_{\uparrow}^{A}=4.4 \pm 0.1 \mathrm{kHz}, \Gamma_{\downarrow}^{A}=2.4 \pm 0.1 \mathrm{kHz}$ and $\Gamma_{\uparrow}^{B}=7.83 \pm 0.05 \mathrm{kHz}, \quad \Gamma_{\downarrow}^{B}=4.64 \pm 0.05 \mathrm{kHz}, \quad$ respectively. The dashed line indicates the duration of the readout pulse. Note that the impact of the readout pulse in this figure is already corrected by the method described in Appendix C 2 .

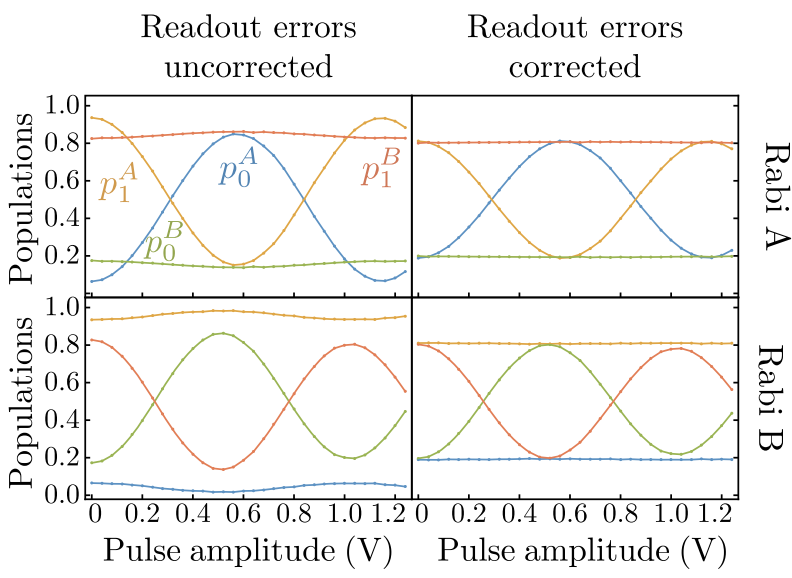

FIG. 10. Rabi oscillations of qubit $A$ (first row) or qubit $B$ (second row) without (left column) and with (right column) readout error correction. After the calibration, only the target qubit displays oscillations.

relaxation rates $\Gamma_{\downarrow}^{A}+\Gamma_{\uparrow}^{A}$ and $\Gamma_{\downarrow}^{B}+\Gamma_{\uparrow}^{B}$ by about a factor of approximately 5 mainly by increasing the excitation rates. The amplitude of the initialization pulse is chosen to maximize the steady state purity while keeping the qubit populations in the computational space. This procedure leads to the preparation of the qubit states in a statistical mixture with the excited state probabilities $p_{1}^{A}=0.86$ and $p_{1}^{B}=0.88$ (see Fig. 11).

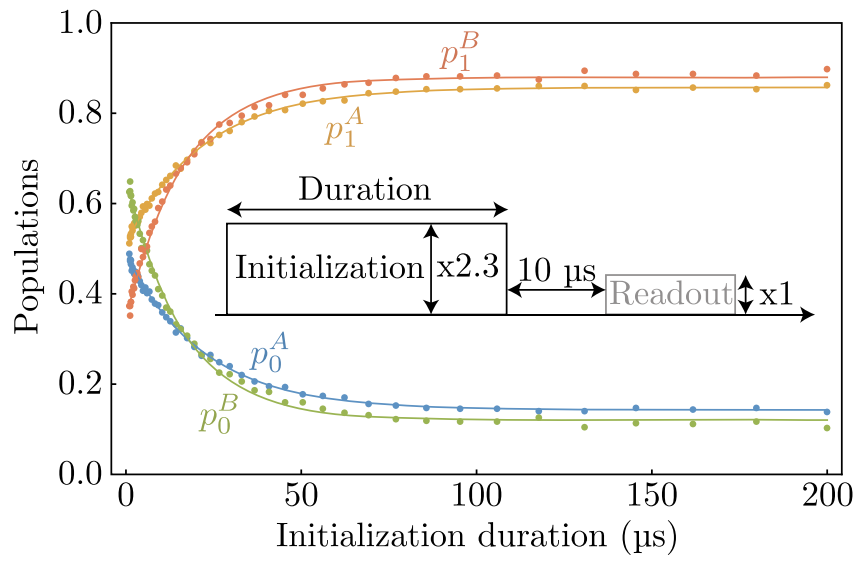

FIG. 11. Initialization of the two fluxoniums. We apply a large (2.3 times the readout amplitude) drive on the cavity in order to reduce the entropy of the system. Populations are extracted by the method described in Appendix C 3. The solid lines correspond to a fit to rate equations for each qubit with the following transition rates: $\Gamma_{\uparrow}^{A}=38.6 \pm 0.6 \mathrm{kHz}, \Gamma_{\downarrow}^{A}=6.4 \pm 0.6 \mathrm{kHz}$ and $\Gamma_{\uparrow}^{B}=53.8 \pm 0.3 \mathrm{kHz}, \Gamma_{\downarrow}^{B}=7.4 \pm 0.3 \mathrm{kHz}$. In all the measurements shown in this paper, we apply a $200 \mu$ s initialization pulse that leaves the qubits in mixed states with excitation probability $p_{1}^{A}=0.86$ and $p_{1}^{B}=0.88$ that correspond to reversed thermal states with temperatures $T_{A}=3.7 \mathrm{mK}$ and $T_{B}=5.9 \mathrm{mK}$. The entropy extracted by the initialization pulse is $\Delta S / \ln (2)=0.81$ bits. 


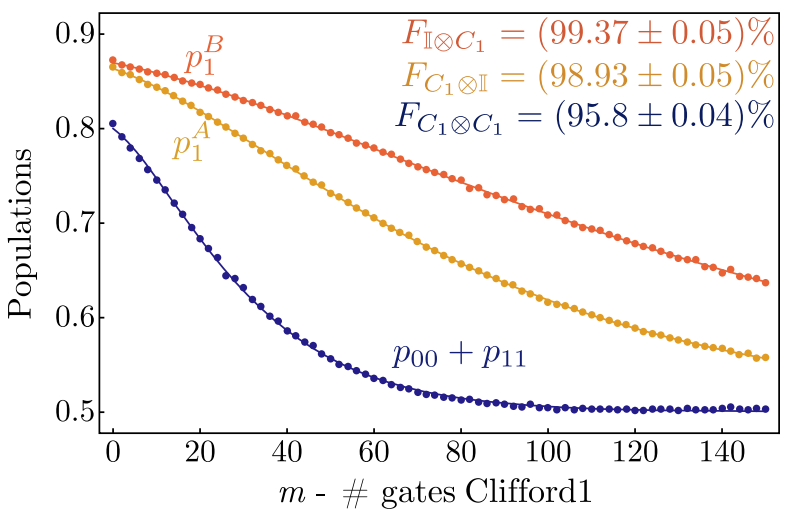

FIG. 12. Randomized benchmarking for single-qubit gates on qubit $A$, qubit $B$, and both. The solid lines are fit by a first-order model [69] $a+b p^{m}+c(m-1) p^{m-2}$, where $p$ is the depolarizing parameter used to calculate the fidelity $F=1-(d-1)$ $(1-p) / d$, where $d$ is the dimension of the Hilbert space. Error bars on the fidelity are calculated according to the procedure described in Appendix E.

\section{APPENDIX D: SINGLE-QUBIT GATE FIDELITIES}

Single-qubit gate fidelities are calibrated by single-qubit randomized benchmarking (see Fig. 12). Qubit $A(B)$ gates are generated using Gaussian edge pulses with a total duration of $150 \mathrm{~ns}$ (75 ns). Reducing the gate duration of single-qubit gates deteriorates the simultaneous randomized benchmarking fidelity because of cross talk.

The addressability of each qubit can be characterized by the numbers in Table III using the definitions in Ref. [62]. The reduction of addressability in our system is related to the fact that our control field aimed at one target qubit is also influencing the other qubit (classical cross talk) due to our choice of a sample design with a single input port.

TABLE III. Table of addressability metrics using the definition of Ref. [62]. The error rates $r_{\alpha}$ are extracted from the independent randomized benchmarking experiments in Fig. 12. The error rates $r_{\alpha \mid \beta}$ are extracted by looking at the depolarization of qubit $\alpha$ during a simultaneous randomized benchmarking experiment. The error metric of error on $\alpha$ due to the unwanted control of $\beta$ is given by $\delta r_{\alpha \mid \beta}=\left|r_{\alpha}-r_{\alpha \mid \beta}\right|$. The correlations in errors are flagged by $\delta p=p_{A B}-p_{A \mid B} p_{B \mid A}$, where $p_{A B}$ is obtained from fitting the decay of qubit-qubit correlations $p_{00}+p_{11}$.

\begin{tabular}{lcc}
\hline \hline Parameter & Twirl group & Experimental value \\
\hline$r_{A}$ & Clifford1 $\otimes \mathbb{I}$ & $6.3 \times 10^{-3}$ \\
$r_{B}$ & $\mathbb{I} \otimes$ Clifford1 & $1.07 \times 10^{-2}$ \\
$r_{A \mid B}$ & Clifford1 $\otimes$ Clifford1 & $5.3 \times 10^{-3}$ \\
$r_{B \mid A}$ & Clifford1 $\otimes$ Clifford1 & $1.4 \times 10^{-2}$ \\
$\delta r_{A \mid B}$ & & $8.1 \times 10^{-3}$ \\
$\delta r_{B \mid A}$ & & $5.4 \times 10^{-3}$ \\
$\delta p$ & & $2.2 \times 10^{-2}$ \\
\hline \hline
\end{tabular}

TABLE IV. Fidelities of various Clifford gates obtained by twoqubit interleaved randomized benchmarking.

\begin{tabular}{lc}
\hline \hline Gate & Fidelity $(\%)$ \\
\hline$X_{\pi / 2}-\mathbb{I}$ & $97.9 \pm 0.1$ \\
$Y_{\pi / 2}-\mathbb{I}$ & $97.9 \pm 0.1$ \\
$X_{\pi}-\mathbb{I}$ & $97.6 \pm 0.1$ \\
$Y_{\pi}-\mathbb{I}$ & $97.6 \pm 0.09$ \\
$\mathbb{I}-X_{\pi / 2}$ & $98.95 \pm 0.08$ \\
$\mathbb{I}-Y_{\pi / 2}$ & $98.96 \pm 0.08$ \\
$\mathbb{I}-X_{\pi}$ & $99.20 \pm 0.08$ \\
$\mathbb{I}-Y_{\pi}$ & $99.20 \pm 0.08$ \\
$X_{\pi / 2}-X_{\pi / 2}$ & $97.8 \pm 0.1$ \\
$X_{\pi / 2}-Y_{\pi / 2}$ & $97.8 \pm 0.1$ \\
$Y_{\pi / 2}-X_{\pi / 2}$ & $97.8 \pm 0.1$ \\
$Y_{\pi / 2}-Y_{\pi / 2}$ & $97.8 \pm 0.1$ \\
$X_{\pi}-X_{\pi}$ & $93.9 \pm 0.2$ \\
$X_{\pi}-Y_{\pi}$ & $94.0 \pm 0.2$ \\
$Y_{\pi}-X_{\pi}$ & $93.8 \pm 0.2$ \\
$Y_{\pi}-Y_{\pi}$ & $94.2 \pm 0.2$ \\
\hline \hline
\end{tabular}

The use of a first-order model [69] to fit the randomized benchmarking decay can be justified by the presence of a time-dependent gate-dependent noise (flux noise) [74]. Table IV summarizes the fidelity of various gates used to generate the Clifford group.

\section{APPENDIX E: ERROR BARS}

The error bars displayed in Fig. 6 are the standard deviations on the population obtained from the population extracted from the method described in Appendix C. All the curves in Fig. 6(a) are fitted simultaneously to the first-order model $a+b p(n)^{m}+c(m-1) p(n)^{m-2}$, where $a, b$, and $c$ are fitting parameters used to absorb SPAM errors, $m$ is the number of Clifford gates, and $n$ is the number of interleaved $\mathrm{CZ}$ gates. The fidelity of an $n$ iteration of a $\mathrm{CZ}$ gate is given by $F\left(\mathrm{CZ}^{n}\right)=1-(d-1)$ $[1-p(n) / p(n=0)] / d$ with $d=4$.

The error on the gate fidelity is then estimated by the propagation of error formula

$\Delta F\left(\mathrm{CZ}^{n}\right)=\frac{d-1}{d} \sqrt{\left(\frac{\Delta p(n)}{p(n=0)}\right)^{2}+\left(\frac{p(n) \Delta p(n=0)}{p(n=0)^{2}}\right)^{2}}$

to obtain the error bars in Fig. 6(b). Similar procedures are used to obtain all the error bars given in the paper.

\section{APPENDIX F: THEORY AND SIMULATIONS OF GATE ERRORS}

\section{Unitary errors}

To study the unitary dynamics during the gate operation, we solve the Schrödinger equation numerically for the 
time-dependent Hamiltonian given by Eqs. (1) and (2) with the additional account for a Gaussian flat-topped pulse in the drive term (2). Thus, we multiply the drive term by a pulse-shaping function with the rising edge given by

$f(t) \propto \exp \left[-\left(t-t_{\text {width }}\right)^{2} / 2 \sigma^{2}\right]-\exp \left[-t_{\text {width }}^{2} / 2 \sigma^{2}\right]$

at $0<t<t_{\text {width }}$, where $\sigma=t_{\text {width }} / \sqrt{2 \pi}$. The lowering edge of the pulse is given by a symmetric expression at $t_{\text {width }}+t_{\text {plateau }}<t<t_{\text {gate }}$, where $t_{\text {plateau }}$ is the duration of the flat part and $t_{\text {gate }}=2 t_{\text {width }}+t_{\text {plateau }}$ is the total gate duration. In all the simulations, we keep $t_{\text {width }}=15 \mathrm{~ns}$ and vary $t_{\text {plateau }}$ in order to vary $t_{\text {gate }}$. To match the measured ratio between resonance Rabi frequencies $\Omega_{10-20}$ and $\Omega_{11-21}$, we choose $\epsilon_{A} / \epsilon_{B}=0.9$ in the drive term (2).

We find the evolution operator $\hat{U}$ by calculating the evolution of four basis computational states, projecting the result into the computational subspace, and performing single-qubit $\mathrm{Z}$ rotations as described in Ref. [25], which ensures that the only diagonal matrix element that has a nonzero phase is $\langle 11|\hat{U}| 11\rangle$. We then calculate the average gate fidelity as [75]

$$
F=\frac{\operatorname{Tr}\left(\hat{U}^{\dagger} \hat{U}\right)+\left|\operatorname{Tr}\left(\hat{U}_{\mathrm{CZ}}^{\dagger} \hat{U}\right)\right|^{2}}{20}
$$

where $\hat{U}_{\mathrm{CZ}}=\operatorname{diag}(1,1,1,-1)$ is the diagonal operator for the ideal $\mathrm{CZ}$ gate. This expression corresponds to the randomized benchmarking fidelity [76]. When the real gate operator is $\hat{U}=\operatorname{diag}\left(1,1,1, e^{i \Delta \varphi}\right)$, we find that $1-F=(3 / 10)(1+\cos \Delta \varphi)$. For a generic gate operator, we define $\Delta \varphi=\arg \langle 11|\hat{U}| 11\rangle$ and use the last expression to calculate the phase error, which is shown by dots in Fig. 3(c). Once projected into the computational subspace, the operator $\hat{U}$ in Eq. (F2) is generally nonunitary, which describes coherent leakage to noncomputational levels. The average probability of such leakage is given by

$$
P_{\text {leak }}=1-\frac{1}{4} \operatorname{Tr}\left(\hat{U}^{\dagger} \hat{U}\right) .
$$

We show this leakage error in Fig. 13(a) as well as by the dashed line in Fig. 3(c). The total gate error calculated using Eq. (F2) is shown in Fig. 13(b) and by the solid line in Fig. 3(c). Results shown in these figures illustrate that we can achieve coherent gate and leakage errors below $10^{-4}$ with the current amplitude and frequency stability of the experiment.

\section{Decoherence effects}

We estimate incoherent errors resulting from driving the $|10\rangle-|20\rangle$ and $|11\rangle-|21\rangle$ transitions as follows. We add the errors coming from cycling each transition independently. We first estimate the state fidelity for an arbitrary
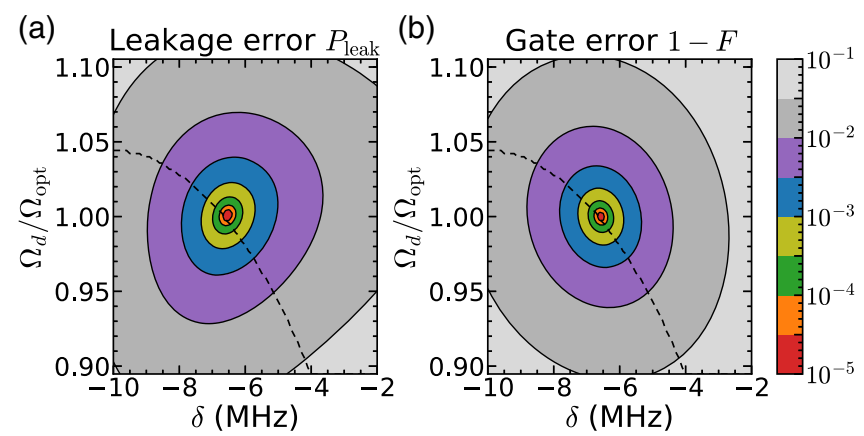

FIG. 13. Simulated leakage error (a) and gate error (b) for the total gate duration of $t_{\text {gate }}=61 \mathrm{~ns}$ versus drive-frequency detuning $\delta$ and drive amplitude $\Omega_{d}$ in units of its optimal value $\Omega_{\text {opt }}$. Dashed lines show parameter values satisfying the correct phase accumulation $\Delta \varphi=\pi$.

initial state when driving a full rotation on one of the transitions and average this fidelity over a set of 36 initial states before summing the two contributions. We solve the master equation by treating incoherent processes as a perturbation and write the density matrix as an expansion $\rho=\rho^{(0)}+\rho^{(1)}+\cdots$. For the initial state $|\psi(0)\rangle=$ $\left|\psi_{\perp}\right\rangle+c|11\rangle$, where $\left|\psi_{\perp}\right\rangle$ describes the amplitudes of the remaining computational basis states, we consider the Hilbert space with only three levels $\left|\psi_{\perp}\right\rangle,|11\rangle$, and $|21\rangle$. Here, $\left\langle\psi_{\perp} \mid \psi_{\perp}\right\rangle=1-|c|^{2}$. We consider the drive Hamiltonian on the $|11\rangle-|21\rangle$ transition in the interaction picture and under the rotating wave approximation

$$
\begin{aligned}
\frac{\hat{H}_{\Omega}}{h} & =\frac{\Omega}{2}(|11\rangle\langle 21|+| 21\rangle\langle 11|) \\
& =\frac{\Omega}{2}|+\rangle\left\langle+\left|-\frac{\Omega}{2}\right|-\right\rangle\langle-|,
\end{aligned}
$$

where we introduce the basis $| \pm\rangle=(|11\rangle \pm|21\rangle) / \sqrt{2}$ that diagonalizes $\hat{H}_{\Omega}$. The unitary evolution under this Hamiltonian results in

$$
\begin{aligned}
|\psi(t)\rangle & =\left|\psi_{\perp}\right\rangle+c[\cos (\pi \Omega t)|11\rangle-i \sin (\pi \Omega t)|21\rangle] \\
& =\left|\psi_{\perp}\right\rangle+c\left[\frac{e^{-i \pi \Omega t}}{\sqrt{2}}|+\rangle+\frac{e^{i \pi \Omega t}}{\sqrt{2}}|-\rangle\right],
\end{aligned}
$$

which gives us the zeroth-order approximation to the density matrix $\rho^{(0)}(t)=|\psi(t)\rangle\langle\psi(t)|$. We find the firstorder correction $\rho^{(1)}(t)$ from the iterative master equation

$$
\frac{d \rho^{(1)}}{d t}=-\frac{i}{\hbar}\left[\hat{H}_{\Omega}, \rho^{(1)}(t)\right]+\mathcal{L} \rho^{(0)}(t),
$$

where the Lindblad superoperator $\mathcal{L} \rho=\sum_{k}\left[\hat{L}_{k} \rho \hat{L}_{k}^{\dagger}-\right.$ $\left.(1 / 2)\left(\hat{L}_{k}^{\dagger} \hat{L}_{k} \rho+\rho \hat{L}_{k}^{\dagger} \hat{L}_{k}\right)\right]$ describes nonunitary processes. We use the following collapse operators: 


$$
\begin{aligned}
& \hat{L}_{1}=\sqrt{\Gamma_{1}}|11\rangle\langle 21|, \\
& \hat{L}_{\varphi}=\sqrt{2 \Gamma_{\varphi}}|21\rangle\langle 21| .
\end{aligned}
$$

The first operator describes relaxation of the $|11\rangle-|21\rangle$ transition with a rate $\Gamma_{1}=1 / T_{1}(11-21)$, and the second operator describes pure dephasing with a rate $\Gamma_{\varphi}=$ $1 / T_{2}^{R}(11-21)-1 / 2 T_{1}(11-21)$ (see Table II).

This result gives the matrix element of the correction in the basis diagonalizing $\hat{H}_{\Omega}$ :

$$
\left\langle m\left|\rho^{(1)}(t)\right| n\right\rangle=\int_{0}^{t}\left\langle m\left|\mathcal{L} \rho^{(0)}(t)\right| n\right\rangle e^{-2 \pi i \nu_{m n}\left(t-t^{\prime}\right)} d t^{\prime},
$$

where $h \nu_{m n}=E_{m}-E_{n}$ is the difference between eigenvalues of $\hat{H}_{\Omega}$ corresponding to $|m\rangle$ and $|n\rangle$. These eigenvalues belong to the set $\{ \pm h \Omega / 2,0\}$.

Using these operators and equations above, we find the following expression for the state-preparation error $\left(t_{\text {gate }}=1 / \Omega\right)$ :

$$
\begin{aligned}
1-F_{\psi}= & -\operatorname{Tr}\left[\rho^{(0)}\left(t_{\text {gate }}\right) \rho^{(1)}\left(t_{\text {gate }}\right)\right] \\
= & \frac{\left(\Gamma_{1}+2 \Gamma_{\varphi}\right) t_{\text {gate }}}{2}|c|^{2}\left(1-|c|^{2}\right) \\
& +\frac{\left(3 \Gamma_{1}+2 \Gamma_{\varphi}\right) t_{\text {gate }}}{8}|c|^{4} .
\end{aligned}
$$

To find the gate error, we average Eq. (F9) over 36 initial two-qubit states generated from single-qubit states $|0\rangle,|1\rangle$, $(|0\rangle \pm|1\rangle) / \sqrt{2}$, and $(|0\rangle \pm i|1\rangle) / \sqrt{2}$. This averaging gives the average values $\left\langle|c|^{2}\right\rangle=1 / 4$ and $\left\langle|c|^{4}\right\rangle=1 / 9$, which results in the gate error

$$
1-F=\frac{\left(2 \Gamma_{1}+3 \Gamma_{\varphi}\right) t_{\text {gate }}}{18}=\frac{1}{36} \frac{t_{\text {gate }}}{T_{1}}+\frac{1}{6} \frac{t_{\text {gate }}}{T_{2}},
$$

where we use the relations $\Gamma_{1}=1 / T_{1}$ and $\Gamma_{\varphi}=1 / T_{2}-$ $1 / 2 T_{1}$. The upper bound for the contribution coming from the $|10\rangle-|20\rangle$ has the same form.

Comparing the values of $T_{1}$ and Ramsey $T_{2}$ in Table II, we find that the main contribution is coming from $T_{2}$, so $1-F \approx t_{\text {gate }} / 6 T_{2}$. Then, for $t_{\text {gate }}=1 / \Delta=45.5 \mathrm{~ns}$ (our analysis is valid for a square pulse), we find that $1-F \approx$ $t_{\text {gate }}\left[1 / 6 T_{2}^{R}(10-20)+1 / 6 T_{2}^{R}(11-21)\right] \approx 0.75 \%$.

We also integrate numerically the master equation in the six-level Hilbert space that consists of the computational subspace and two upper levels $|20\rangle$ and $|21\rangle$. We consider the Hamiltonian generalized from Eq. (F4) to include the drive of the $|10\rangle-|20\rangle$ transition and to account for detunings between the drive frequency and two transition frequencies as described in Sec. III. Using collapse operators given in Eqs. (F7a) and (F7b) and similar operators for the $|10\rangle-|20\rangle$ transition, we find the incoherent error to be $0.62 \%$, which is slightly smaller than the analytic estimate of $0.75 \%$ because of the proper account for the drivefrequency detuning. Including additional single-qubit relaxation and dephasing channels with Ramsey $T_{2}$, we find the upper numerical bound for the incoherent error to be $0.95 \%$.

[1] M. Kjaergaard, M.E. Schwartz, J. Braumüller, P. Krantz, J. I. Wang, S. Gustavsson, and W. D. Oliver, Superconducting Qubits: Current State of Play, Annu. Rev. Condens. Matter Phys. 11, 369 (2020).

[2] J. Kelly et al., State Preservation by Repetitive Error Detection in a Superconducting Quantum Circuit, Nature (London) 519, 66 (2015).

[3] J. S. Otterbach et al., Unsupervised Machine Learning on a Hybrid Quantum Computer, arXiv:1712.05771.

[4] A. Kandala, A. Mezzacapo, K. Temme, M. Takita, M. Brink, J. M. Chow, and J. M. Gambetta, Hardware-Efficient Variational Quantum Eigensolver for Small Molecules and Quantum Magnets, Nature (London) 549, 242 (2017).

[5] C. Song, K. Xu, W. Liu, C. P. Yang, S. B. Zheng, H. Deng, Q. Xie, K. Huang, Q. Guo, L. Zhang, P. Zhang, D. Xu, D. Zheng, X. Zhu, H. Wang, Y. A. Chen, C. Y. Lu, S. Han, and J. W. Pan, 10-Qubit Entanglement and Parallel Logic Operations with a Superconducting Circuit, Phys. Rev. Lett. 119, 180511 (2017).

[6] C. Neill et al., A Blueprint for Demonstrating Quantum Supremacy with Superconducting Qubits, Science 360, 195 (2018).

[7] K. X. Wei, I. Lauer, S. Srinivasan, N. Sundaresan, D. T. McClure, D. Toyli, D. C. Mckay, J. M. Gambetta, and S. Sheldon, Verifying Multipartite Entangled GreenbergerHorne-Zeilinger States via Multiple Quantum Coherences, Phys. Rev. A 101, 032343 (2020).

[8] P. Jurcevic et al., Demonstration of Quantum Volume 64 on a Superconducting Quantum Computing System, arXiv: 2008.08571.

[9] A. Hashim, R. K. Naik, A. Morvan, J.-L. Ville, B. Mitchell, J. M. Kreikebaum, M. Davis, E. Smith, C. Iancu, K. P. O'Brien, I. Hincks, J. J. Wallman, J. Emerson, and I. Siddiqi, Randomized Compiling for Scalable Quantum Computing on a Noisy Superconducting Quantum Processor, arXiv:2010.00215.

[10] N. Ofek, A. Petrenko, R. Heeres, P. Reinhold, Z. Leghtas, B. Vlastakis, Y. Liu, L. Frunzio, S. M. Girvin, L. Jiang, M. Mirrahimi, M. H. Devoret, and R. J. Schoelkopf, Extending the Lifetime of a Quantum Bit with Error Correction in Superconducting Circuits, Nature (London) 536, 441 (2016).

[11] U. L. Heras, A. Mezzacapo, L. Lamata, S. Filipp, A. Wallraff, and E. Solano, Digital Quantum Simulation of Spin Systems in Superconducting Circuits, Phys. Rev. Lett. 112, 200501 (2014).

[12] Y. Salathé, M. Mondal, M. Oppliger, J. Heinsoo, P. Kurpiers, A. Potočnik, A. Mezzacapo, U. Las Heras, L. Lamata, E. Solano, S. Filipp, and A. Wallraff, Digital Quantum Simulation of Spin Models with Circuit Quantum Electrodynamics, Phys. Rev. X 5, 021027 (2015). 
[13] R. Barends et al., Digitized Adiabatic Quantum Computing with a Superconducting Circuit, Nature (London) 534, 222 (2016).

[14] F. Arute et al., Observation of Separated Dynamics of Charge and Spin in the Fermi-Hubbard Model, arXiv: 2010.07965.

[15] D. A. Fedorov, M. J. Otten, S. K. Gray, and Y. Alexeev, Ab Initio Molecular Dynamics on Quantum Computers, arXiv:2008.06562.

[16] B. Fauseweh and J.-X. Zhu, Digital Quantum Simulation of Non-Equilibrium Quantum Many-Body Systems, arXiv: 2009.07375.

[17] R. Barends et al., Digital Quantum Simulation of Fermionic Models with a Superconducting Circuit, Nat. Commun. 6, 7654 (2015).

[18] N. Moll, P. Barkoutsos, L. S. Bishop, J. M. Chow, A. Cross, D. J. Egger, S. Filipp, A. Fuhrer, J. M. Gambetta, M. Ganzhorn, A. Kandala, A. Mezzacapo, P. Müller, W. Riess, G. Salis, J. Smolin, I. Tavernelli, and K. Temme, Quantum Optimization Using Variational Algorithms on Near-Term Quantum Devices, Quantum Sci. Technol. 3, 030503 (2018).

[19] M. P. Harrigan et al., Quantum Approximate Optimization of Non-Planar Graph Problems on a Planar Superconducting Processor, Nat. Phys. 17, 332 (2021).

[20] F. Arute et al., Quantum Supremacy Using a Programmable Superconducting Processor, Nature (London) 574, 505 (2019).

[21] J. Koch, T. M. Yu, J. Gambetta, A. A. Houck, D. I. Schuster, J. Majer, A. Blais, M. H. Devoret, S. M. Girvin, and R. J. Schoelkopf, Charge-Insensitive Qubit Design Derived from the Cooper Pair Box, Phys. Rev. A 76, 042319 (2007).

[22] L. B. Nguyen, Y. H. Lin, A. Somoroff, R. Mencia, N. Grabon, and V. E. Manucharyan, High-Coherence Fluxonium Qubit, Phys. Rev. X 9, 041041 (2019).

[23] H. Zhang, S. Chakram, T. Roy, N. Earnest, Y. Lu, Z. Huang, D. Weiss, J. Koch, and D. I. Schuster, Universal Fast Flux Control of a Coherent, Low-Frequency Qubit, Phys. Rev. X 11, 011010 (2021).

[24] V. E. Manucharyan, J. Koch, L. I. Glazman, and M. H. Devoret, Fluxonium: Single Cooper-Pair Circuit Free of Charge Offsets, Science 326, 113 (2009).

[25] K. N. Nesterov, I. V. Pechenezhskiy, C. Wang, V.E. Manucharyan, and M.G. Vavilov, Microwave-Activated Controlled-Z Gate for Fixed-Frequency Fluxonium Qubits, Phys. Rev. A 98, 030301(R) (2018).

[26] A. A. Abdumalikov, J. M. Fink, K. Juliusson, M. Pechal, S. Berger, A. Wallraff, and S. Filipp, Experimental Realization of Non-Abelian Non-adiabatic Geometric Gates, Nature (London) 496, 482 (2013).

[27] D. J. Egger and F. K. Wilhelm, Optimized Controlled-Z Gates for Two Superconducting Qubits Coupled through a Resonator, Supercond. Sci. Technol. 27, 014001 (2014).

[28] R. Barends et al., Diabatic Gates for Frequency-Tunable Superconducting Qubits, Phys. Rev. Lett. 123, 210501 (2019).

[29] S. Krinner, P. Kurpiers, B. Royer, P. Magnard, I. Tsitsilin, J. C. Besse, A. Remm, A. Blais, and A. Wallraff, Demonstration of an All-Microwave Controlled-Phase Gate between Far Detuned Qubits, Phys. Rev. Applied 14, 044039 (2020).

[30] M. Ganzhorn, G. Salis, D. J. Egger, A. Fuhrer, M. Mergenthaler, C. Müller, P. Müller, S. Paredes, M. Pechal, M. Werninghaus, and S. Filipp, Benchmarking the Noise Sensitivity of Different Parametric Two-Qubit Gates in a Single Superconducting Quantum Computing Platform, Phys. Rev. Research 2, 033447 (2020).

[31] V. Negîrneac, H. Ali, N. Muthusubramanian, F. Battistel, R. Sagastizabal, M. S. Moreira, J. F. Marques, W. Vlothuizen, M. Beekman, N. Haider, A. Bruno, and L. DiCarlo, HighFidelity Controlled-Z Gate with Maximal Intermediate Leakage Operating at the Speed Limit in a Superconducting Quantum Processor, arXiv:2008.07411.

[32] F. W. Strauch, P. R. Johnson, A. J. Dragt, C. J. Lobb, J. R. Anderson, and F. C. Wellstood, Quantum Logic Gates for Coupled Superconducting Phase Qubits, Phys. Rev. Lett. 91, 167005 (2003).

[33] L. Dicarlo, J. M. Chow, J. M. Gambetta, L. S. Bishop, B. R. Johnson, D. I. Schuster, J. Majer, A. Blais, L. Frunzio, S. M. Girvin, and R. J. Schoelkopf, Demonstration of Two-Qubit Algorithms with a Superconducting Quantum Processor, Nature (London) 460, 240 (2009).

[34] J. M. Chow, J. M. Gambetta, A. W. Cross, S. T. Merkel, C. Rigetti, and M. Steffen, Microwave-Activated ConditionalPhase Gate for Superconducting Qubits, New J. Phys. 15, 115012 (2013).

[35] A. Blais, A. M. van den Brink, and A. M. Zagoskin, Tunable Coupling of Superconducting Qubits, Phys. Rev. Lett. 90, 127901 (2003).

[36] R. C. Bialczak, M. Ansmann, M. Hofheinz, E. Lucero, M. Neeley, A. D. Oconnell, D. Sank, H. Wang, J. Wenner, M. Steffen, A. N. Cleland, and J. M. Martinis, Quantum Process Tomography of a Universal Entangling Gate Implemented with Josephson Phase Qubits, Nat. Phys. 6, 409 (2010).

[37] A. Dewes, F. R. Ong, V. Schmitt, R. Lauro, N. Boulant, P. Bertet, D. Vion, and D. Esteve, Characterization of a TwoTransmon Processor with Individual Single-Shot Qubit Readout, Phys. Rev. Lett. 108, 057002 (2012).

[38] C. Rigetti and M. Devoret, Fully Microwave-Tunable Universal Gates in Superconducting Qubits with Linear Couplings and Fixed Transition Frequencies, Phys. Rev. B 81, 134507 (2010).

[39] J. M. Chow, A. D. Córcoles, J. M. Gambetta, C. Rigetti, B. R. Johnson, J. A. Smolin, J. R. Rozen, G. A. Keefe, M. B. Rothwell, M. B. Ketchen, and M. Steffen, Simple AllMicrowave Entangling Gate for Fixed-Frequency Superconducting Qubits, Phys. Rev. Lett. 107, 080502 (2011).

[40] S. Sheldon, E. Magesan, J. M. Chow, and J. M. Gambetta, Procedure for Systematically Tuning up Cross-Talk in the Cross-Resonance Gate, Phys. Rev. A 93, 060302(R) (2016).

[41] N. Sundaresan, I. Lauer, E. Pritchett, E. Magesan, P. Jurcevic, and J. M. Gambetta, Reducing Unitary and Spectator Errors in Cross Resonance with Optimized Rotary Echoes, PRX Quantum 1, 020318 (2020).

[42] P. Mundada, G. Zhang, T. Hazard, and A. Houck, Suppression of Qubit Crosstalk in a Tunable Coupling Superconducting Circuit, Phys. Rev. Applied 12, 054023 (2019). 
[43] X. Li, T. Cai, H. Yan, Z. Wang, X. Pan, Y. Ma, W. Cai, J. Han, Z. Hua, X. Han, Y. Wu, H. Zhang, H. Wang, Y. Song, L. Duan, and L. Sun, Tunable Coupler for Realizing a Controlled-Phase Gate with Dynamically Decoupled Regime in a Superconducting Circuit, Phys. Rev. Applied 14, 024070 (2020).

[44] A. Noguchi, A. Osada, S. Masuda, S. Kono, K. Heya, S. P. Wolski, H. Takahashi, T. Sugiyama, D. Lachance-Quirion, and Y. Nakamura, Fast Parametric Two-Qubit Gates with Suppressed Residual Interaction Using the Second-Order Nonlinearity of a Cubic Transmon, Phys. Rev. A 102, 062408 (2020).

[45] D. C. McKay, S. Sheldon, J. A. Smolin, J. M. Chow, and J. M. Gambetta, Three-Qubit Randomized Benchmarking, Phys. Rev. Lett. 122, 200502 (2019).

[46] A. Kandala, K. X. Wei, S. Srinivasan, E. Magesan, S. Carnevale, G. A. Keefe, D. Klaus, O. Dial, and D. C. McKay, Demonstration of a High-Fidelity CNOTfor Fixed-Frequency Transmons with Engineered ZZ Suppression, arXiv:2011 .07050 .

[47] A. D. Córcoles, J. M. Gambetta, J. M. Chow, J. A. Smolin, M. Ware, J. Strand, B. L. T. Plourde, and M. Steffen, Process Verification of Two-Qubit Quantum Gates by Randomized Benchmarking, Phys. Rev. A 87, 030301(R) (2013).

[48] D. I. Schuster, A. Wallraff, A. Blais, L. Frunzio, R. S. Huang, J. Majer, S. M. Girvin, and R. J. Schoelkopf, Ac Stark Shift and Dephasing of a Superconducting Qubit Strongly Coupled to a Cavity Field, Phys. Rev. Lett. 94, 123602 (2005).

[49] V. E. Manucharyan, J. Koch, M. Brink, L. I. Glazman, and M. H. Devoret, Coherent Oscillations between Classically Separable Quantum States of a Superconducting Loop, arXiv:0910.3039.

[50] R. Gebauer, N. Karcher, D. Gusenkova, M. Spiecker, L. Grünhaupt, I. Takmakov, P. Winkel, L. Planat, N. Roch, W. Wernsdorfer, A. V. Ustinov, M. Weber, M. Weides, I. M. Pop, and O. Sander, State Preparation of a Fluxonium Qubit with Feedback from a Custom FPGA-Based Platform, AIP Conf. Proc. 2241, 020015 (2020).

[51] C. Macklin, D. Hover, M. E. Schwartz, X. Zhang, W. D. Oliver, and I. Siddiqi, A Near-Quantum-Limited Josephson Traveling-Wave Parametric Amplifier, Science 350, 307 (2015).

[52] D. C. McKay, C. J. Wood, S. Sheldon, J. M. Chow, and J. M. Gambetta, Efficient Z Gates for Quantum Computing, Phys. Rev. A 96, 022330 (2017).

[53] T. Roy, S. Hazra, S. Kundu, M. Chand, M. P. Patankar, and R. Vijay, Programmable Superconducting Processor with Native Three-Qubit Gates, Phys. Rev. Applied 14, 014072 (2020).

[54] M. A. Nielsen and I. L. Chuang, Quantum Computation and Quantum Information, 1st ed. (Cambridge University Press, 2010).

[55] J. M. Chow, J. M. Gambetta, A. D. Córcoles, S. T. Merkel, J. A. Smolin, C. Rigetti, S. Poletto, G. A. Keefe, M. B. Rothwell, J. R. Rozen, M. B. Ketchen, and M. Steffen, Universal Quantum Gate Set Approaching Fault-Tolerant Thresholds with Superconducting Qubits, Phys. Rev. Lett. 109, 060501 (2012).

[56] E. Magesan, J. M. Gambetta, and J. Emerson, Characterizing Quantum Gates via Randomized Benchmarking, Phys. Rev. A 85, 042311 (2012).
[57] J. Kelly, R. Barends, B. Campbell, Y. Chen, Z. Chen, B. Chiaro, A. Dunsworth, A. G. Fowler, I. C. Hoi, E. Jeffrey, A. Megrant, J. Mutus, C. Neill, P. J. J. O’Malley, C. Quintana, P. Roushan, D. Sank, A. Vainsencher, J. Wenner, T. C. White, A. N. Cleland, and J. M. Martinis, Optimal Quantum Control Using Randomized Benchmarking, Phys. Rev. Lett. 112, 240504 (2014).

[58] N. Hansen, Y. Akimoto, and P. Baudis, CMA-ES/pycma, https://doi.org/DOI:10.5281/zenodo.2559634.

[59] S. Sheldon, L. S. Bishop, E. Magesan, S. Filipp, J. M. Chow, and J. M. Gambetta, Characterizing Errors on Qubit Operations via Iterative Randomized Benchmarking, Phys. Rev. A 93, 012301 (2016).

[60] Z. Wang, S. Shankar, Z. K. Minev, P. Campagne-Ibarcq, A. Narla, and M. H. Devoret, Cavity Attenuators for Superconducting Qubits, Phys. Rev. Applied 11, 014031 (2019).

[61] F. Yan, D. Campbell, P. Krantz, M. Kjaergaard, D. Kim, J. L. Yoder, D. Hover, A. Sears, A. J. Kerman, T. P. Orlando, S. Gustavsson, and W. D. Oliver, Distinguishing Coherent and Thermal Photon Noise in a Circuit Quantum Electrodynamical System, Phys. Rev. Lett. 120, 260504 (2018).

[62] J. M. Gambetta, A. D. Córcoles, S. T. Merkel, B. R. Johnson, J. A. Smolin, J. M. Chow, C. A. Ryan, C. Rigetti, S. Poletto, T. A. Ohki, M. B. Ketchen, and M. Steffen, Characterization of Addressability by Simultaneous Randomized Benchmarking, Phys. Rev. Lett. 109, 240504 (2012).

[63] V. Tripathi, M. Khezri, and A. N. Korotkov, Operation and Intrinsic Error Budget of a Two-Qubit Cross-Resonance Gate, Phys. Rev. A 100, 012301 (2019).

[64] S. Filipp, P. Maurer, P. J. Leek, M. Baur, R. Bianchetti, J. M. Fink, M. Göppl, L. Steffen, J. M. Gambetta, A. Blais, and A. Wallraff, Two-Qubit State Tomography Using a Joint Dispersive Readout, Phys. Rev. Lett. 102, 200402 (2009).

[65] G. Zhu, D. G. Ferguson, V. E. Manucharyan, and J. Koch, Circuit QED with Fluxonium Qubits: Theory of the Dispersive Regime, Phys. Rev. B 87, 024510 (2013).

[66] M. Boissonneault, J. M. Gambetta, and A. Blais, Dispersive Regime of Circuit QED: Photon-Dependent Qubit Dephasing and Relaxation Rates, Phys. Rev. A 79, 013819 (2009).

[67] D. H. Slichter, R. Vijay, S. J. Weber, S. Boutin, M. Boissonneault, J. M. Gambetta, A. Blais, and I. Siddiqi, Measurement-Induced Qubit State Mixing in Circuit QED from Up-Converted Dephasing Noise, Phys. Rev. Lett. 109, 153601 (2012).

[68] D. Sank et al., Measurement-Induced State Transitions in a Superconducting Qubit: Beyond the Rotating Wave Approximation, Phys. Rev. Lett. 117, 190503 (2016).

[69] E. Magesan, J. M. Gambetta, B. R. Johnson, C. A. Ryan, J. M. Chow, S. T. Merkel, M. P. da Silva, G. A. Keefe, M. B. Rothwell, T. A. Ohki, M. B. Ketchen, and M. Steffen, Efficient Measurement of Quantum Gate Error by Interleaved Randomized Benchmarking, Phys. Rev. Lett. 109, 080505 (2012).

[70] M. D. Reed, B. R. Johnson, A. A. Houck, L. Dicarlo, J. M. Chow, D. I. Schuster, L. Frunzio, and R. J. Schoelkopf, Fast Reset and Suppressing Spontaneous Emission of a Superconducting Qubit, Appl. Phys. Lett. 96, 203110 (2010).

[71] P. Magnard, P. Kurpiers, B. Royer, T. Walter, J. C. Besse, S. Gasparinetti, M. Pechal, J. Heinsoo, S. Storz, A. Blais, and A. Wallraff, Fast and Unconditional All-Microwave Reset of 
a Superconducting Qubit, Phys. Rev. Lett. 121, 060502 (2018).

[72] V. E. Manucharyan, Superinductance, Ph. D. thesis, Yale Univeristy, 2012.

[73] D. Gusenkova, M. Spiecker, R. Gebauer, M. Willsch, F. Valenti, N. Karcher, L. Grünhaupt, I. Takmakov, P. Winkel, D. Rieger, A. V. Ustinov, N. Roch, W. Wernsdorfer, K. Michielsen, O. Sander, and I. M. Pop, Quantum Nondemolition Dispersive Readout of a Superconducting Artificial Atom Using Large Photon Numbers, arXiv:2009.14785.
[74] J. J. Wallman, Randomized Benchmarking with GateDependent Noise, Quantum 2, 47 (2018).

[75] L. H. Pedersen, N. M. Møller, and K. Mølmer, Fidelity of Quantum Operations, Phys. Lett. A 367, 47 (2007).

[76] J. M. Chow, J. M. Gambetta, L. Tornberg, J. Koch, L. S. Bishop, A. A. Houck, B. R. Johnson, L. Frunzio, S. M. Girvin, and R. J. Schoelkopf, Randomized Benchmarking and Process Tomography for Gate Errors in a Solid-State Qubit, Phys. Rev. Lett. 102, 090502 (2009). 